\title{
First variation of anisotropic energies and crystalline mean curvature for partitions
}

\author{
G. BELlETTINI ${ }^{\dagger}$ AND G. RIEY \\ Dipartimento di Matematica, Università di Roma "Tor Vergata" \\ via della Ricerca Scientifica, 00133 Roma, Italy \\ AND \\ M. NovaGA ${ }^{\S}$ \\ Dipartimento di Matematica, Università di Pisa \\ via Buonarroti 2, 56127 Pisa, Italy
}

[Received 7 November 2002]

\begin{abstract}
We rigorously derive the notion of crystalline mean curvature of an anisotropic partition with no restriction on the space dimension. Our results cover the case of crystalline networks in two dimensions, polyhedral partitions in three dimensions, and generic anisotropic partitions for smooth anisotropies. The natural equilibrium conditions on the singular set of the partition are derived. We discuss several examples in two dimensions (also for two adjacent triple junctions) and one example in three dimensions when the Wulff shape is the unit cube. In the examples we also analyze the stability of the partitions.
\end{abstract}

\section{Introduction}

The study of the properties of polycrystalline materials is an important field of research in material science and in chemistry; in particular, understanding the geometry and stability of triple (and more generally multiple) junctions of interfaces is of interest in microstructures and in the evolution of grain boundaries [8], [11], [17], [23], [21], [10].

In this paper we are interested in deriving rigorously the notion of crystalline mean curvature of an anisotropic partition with no restriction on the space dimension. Our study includes crystalline networks in two dimensions and polyhedral partitions in three-dimensional space; we can also treat an arbitrary smooth anisotropy. As a by-product of our results, we derive the necessary conditions that multiple junctions must satisfy in order to be an equilibrium configuration. We also uniquely find the velocity field which is expected to drive the subsequent evolution process.

From the mathematical point of view, the first definitions and results on crystalline geometry were given by J. E. Taylor in several papers (see for instance [24], [27], [9], [26], [28]). In these papers the author defines crystalline mean curvature for a polygonal curve and for a network of curves, looking at the rate of change of the total free energy surface with volume swept under deformations. In this way the crystalline curvature flow for a polygonal curve is derived, as well as the motion of networks and triple junctions. A rather interesting discussion on whether (and

\footnotetext{
†Email: belletti@mat.uniroma2.it

‡Email: riey@mat.uniroma2.it

$\S$ Email: novaga@dm.unipi.it
} 
how) additional line segments have to be added at triple junctions to decrease the total energy is outlined in [28]; the utility of this issue relies once more on the associated evolution process. As we shall see, in three dimensions the situation is much more difficult; we refer to [25], [27], [22], [3], [4] for some results in this direction. Concerning other results on anisotropic partitions and related evolution problems, we refer to [14], [13], [15], [20].

Our approach is based on ideas different from those in the above cited papers, and relies in particular on the theory outlined in [3] where, through the first variation of the total energy, the crystalline (or, in general, anisotropic) mean curvature $\kappa_{\varphi}$ is computed for boundaries of sets (i.e. when only two phases are present). Some by-products of those computations, which are performed in any dimension, are: (i) a (pointwise) definition of $\kappa_{\varphi}$ as the unique solution of a variational problem; (ii) $L^{\infty}$ and $B V$-regularity of $\kappa_{\varphi}$; (iii) the facet breaking/bending phenomena (for the related flow) in connection with the regularity of $\kappa_{\varphi}$.

Following those ideas, in order to derive the anisotropic mean curvature of a partition (i.e., when at least three different phases are present) it is natural to compute the first variation of the energy, now defined as the Minkowski content $\mathcal{M}_{\varphi}$ of the whole interface in the relative geometry induced by the anisotropy $\varphi$ itself.

Beside the usual difficulties (i.e., the nonsmoothness both of the interface and of the density of energy, see [3]), we must face further difficulties due to the fact that now we cannot restrict the variation to $\varphi$-normal vector fields; indeed tangential components cannot be neglected, especially in a neighbourhood of the singular set.

Let us briefly explain the content of the paper and the main results. After introducing the notation (Section 2), we begin by computing the first variation of the energy in the smooth case, i.e. when the anisotropy is strictly convex and smooth (Section 3). We perform the first variation first in two dimensions using a parametric approach (Theorem 3.4) and then in any dimension (Theorem 3.6). These computations could be of some interest from the point of view of Finsler geometry, since they are based on an integration by parts formula on manifolds with boundary (formula (14). Furthermore, these results are enlightening in order to approach the crystalline case. One observation of this section is of particular interest: given a manifold $\Sigma \subset \mathbb{R}^{n}$ with boundary, we can define the analog of the unit conormal vector field $n_{\varphi}^{\partial \Sigma}$ on the boundary of $\Sigma$, in the geometry induced by the anisotropy (see the last part of the proof of Theorem 3.6 and Definition 3.1). This vector field is constructed starting from the intrinsic unit vector field $n_{\varphi}$ to $\Sigma$ (sometimes called the Cahn-Hoffman field [19]). More precisely, $n_{\varphi}^{\partial \Sigma}$ turns out to be, on $\partial \Sigma$, the component of $n_{\varphi}^{\partial \Sigma}$ in the normal space to $\partial \Sigma$ rotated through $\pi / 2$ in such a way that $n_{\varphi}^{\partial \Sigma}$ points outwards from $\Sigma$. It is through $n_{\varphi}^{\partial \Sigma}$ that the equilibrium condition at the junction can be expressed in any dimension (see (24)). Such an equilibrium condition is (locally near the singular set) equivalent to the usual force balance (also called Young's law or Herring condition [18], [19], [22]).

In Section 4 we focus our attention on the nonsmooth, in particular crystalline, case. To make the computations rigorous, we need to introduce several definitions, which resemble those given in [3] for the two-phases case.

The main result of the paper is contained in Theorem 4.8, roughly speaking, it turns out that the (uniquely determined) mean curvature $\kappa_{\varphi}$ of a crystalline partition $T$ is the tangential divergence of a vector field $N_{\min }$ which minimizes the functional

$$
\int_{T}\left(\operatorname{div}_{\tau} N\right)^{2} \mathrm{~d} \mathcal{P}_{\varphi}
$$


among all Cahn-Hoffman vector fields $N \in H_{v, \varphi}^{\mathrm{div}}\left(T ; \mathbb{R}^{n}\right)$ satisfying the condition $\sum_{i, j} \widehat{N}^{\partial \Sigma_{i j}}=0$ on the singular set common to all the "sheets" $\Sigma_{i j}$ of $T$. Here d $\mathcal{P}_{\varphi}$ is the density of the Minkowski content, which is expressed in a natural way through the dual norm $\varphi^{o}$ (surface tension) of $\varphi$ (see 5). The symbol $H_{v, \varphi}^{\text {div }}\left(T ; \mathbb{R}^{n}\right)$ denotes the space where the functional 1 is naturally defined, i.e. the space of all $\varphi$-normal vector fields whose restriction to each $\Sigma_{i j}$ has square integrable divergence. Finally, $\widehat{N}^{\partial \Sigma_{i j}}$ is a suitable $\pi / 2$ rotation of a well defined trace $\widehat{N}$ of $N$ on $\partial \Sigma_{i j}$ (see Definition 4.4.

As shown in Section 5 , in a number of situations the minimum problem (1) can be made explicit and its solution explicitly computed. For instance, in the two-dimensional crystalline case and for certain three-dimensional partitions, the functional (1) reduces to a quadratic polynomial in a finite number of variables, to be minimized on a compact domain. This observation allows us to compute the pointwise crystalline mean curvature for many (possibly adjacent) triple or multiple junctions in two dimensions as well as for a partition in three dimensions. Of course, the function to be minimized can be quite involved, as for instance for two or more than two adjacent triple junctions in a network. We show here explicit computations in two dimensions when the Wulff shape is an octagon (see Examples 1, 2, 3, and Examples 4 and 5 for two adjacent triple junctions) and in three dimensions when the Wulff shape is a cube (see subsection 5.2. In two dimensions, we discuss the stability of triple junctions, in connection with the related evolution process. We show that some triple junctions are always unstable (Example 3), as are suitable adjacent triple junctions (Example 5).

Finally, our results give a unique velocity field in the associated evolution process (the anisotropic mean curvature flow of the partition) and indicate, in two dimensions, the nature of the process leading to the creation of new edges at a triple junction (in agreement with the observations in [28]); see the discussion in Example 1. In three dimensions far more complicated behaviours are expected, beside the facet breaking/bending phenomena observed in the two-phases case [3].

Using our approach, in a subsequent paper [5] we shall investigate the local existence and uniqueness of the crystalline flow for a partition in two dimensions.

\section{Notation}

In the following we denote by $\cdot$ the standard euclidean scalar product in $\mathbb{R}^{n}$ and by $|\cdot|$ the euclidean norm in $\mathbb{R}^{n}, n \geqslant 2$. Given two vectors $a, b \in \mathbb{R}^{n}$, we denote by $a \otimes b$ the matrix whose entries are $(a \otimes b)_{i j}:=a_{i} b_{j}$. The symbol $\mathcal{H}^{k}$ denotes the $k$-dimensional Hausdorff measure in $\mathbb{R}^{n}, k \in[0, n]$. Given a linear subspace $V \subseteq \mathbb{R}^{n}$ we denote by $V^{\perp}$ the orthogonal complement of $V$. Given a vector $v \in \mathbb{R}^{2}$, we denote by $v^{\perp}$ the rotation of $v$ through $\pi / 2$ around the origin in counterclockwise direction.

\subsection{Finsler norms}

We denote by $\varphi: \mathbb{R}^{n} \rightarrow\left[0,+\infty\left[\right.\right.$ a Finsler norm on $\mathbb{R}^{n}$, i.e. a convex function satisfying

$$
\varphi(\lambda \xi)=|\lambda| \varphi(\xi), \quad \varphi(\xi) \geqslant c|\xi|, \quad \lambda \in \mathbb{R}, \xi \in \mathbb{R}^{n},
$$

for some $c>0$. We define

- the dual $\varphi^{o}: \mathbb{R}^{n} \rightarrow\left[0,+\infty\left[\right.\right.$ of $\varphi, \varphi^{o}\left(\xi^{o}\right):=\sup \left\{\xi \cdot \xi^{o}: \varphi(\xi) \leqslant 1\right\}$ for any $\xi^{o} \in \mathbb{R}^{n}$;

- the unit ball $\mathcal{W}_{\varphi}:=\left\{\xi \in \mathbb{R}^{n}: \varphi(\xi) \leqslant 1\right\}$, sometimes called the Wulff shape;

- the unit ball $\mathcal{F}_{\varphi}:=\left\{\xi^{o} \in \mathbb{R}^{n}: \varphi^{o}\left(\xi^{o}\right) \leqslant 1\right\}$, sometimes called the Frank diagram; 
- the duality mappings

$$
\begin{aligned}
& T(\xi):=\left\{\xi^{o} \in \mathbb{R}^{n}: \xi^{o} \cdot \xi=\varphi(\xi)^{2}=\varphi^{o}\left(\xi^{o}\right)^{2}\right\}=\frac{1}{2} \partial\left(\varphi(\xi)^{2}\right), \quad \xi \in \mathbb{R}^{n}, \\
& T^{o}\left(\xi^{o}\right):=\left\{\xi \in \mathbb{R}^{n}: \xi \cdot \xi^{o}=\varphi^{o}\left(\xi^{o}\right)^{2}=\varphi(\xi)^{2}\right\}=\frac{1}{2} \partial\left(\varphi^{o}\left(\xi^{o}\right)^{2}\right), \quad \xi^{o} \in \mathbb{R}^{n},
\end{aligned}
$$

$\partial$ denoting the usual subdifferential for convex functions.

We say that $\varphi$ is smooth if $\mathcal{W}_{\varphi}$ and $\mathcal{F}_{\varphi}$ are two strictly convex bodies with smooth boundary. We say that $\varphi$ is crystalline if $\mathcal{W}_{\varphi}$ is a convex polytope.

Concerning the relations between the above definitions and anisotropic and crystalline motion by mean curvature we refer for instance to [6], [3], and references therein.

\subsection{Lipschitz hypersurfaces with boundary. The Minkowski content}

By a Lipschitz hypersurface with (Lipschitz) boundary we mean an $(n-1)$-dimensional bounded set $\Sigma \subset \mathbb{R}^{n}$ which can be written locally as a Lipschitz graph on an open set, and such that each point of its boundary can be written locally as a Lipschitz graph on an open Lipschitz subset of $\mathbb{R}^{n-1}$. If $x \in \Sigma$ (resp. $x \in \partial \Sigma$ ) we denote by $T_{x}(\Sigma)$ (resp. $T_{x}(\partial \Sigma)$ ) the tangent space to $\Sigma$ (resp. to $\partial \Sigma$ ) at $x$. We also denote by $\Pi_{T_{x}(\Sigma)}$ (resp. $\Pi_{T_{x}(\partial \Sigma)}$ ) the orthogonal projection on $T_{x}(\Sigma)$ (resp. on $T_{x}(\partial \Sigma)$ ). If $g: \Sigma \rightarrow \mathbb{R}^{n}$ is a Lipschitz vector field, we denote by $\operatorname{div}_{\tau} g$ the euclidean tangential divergence of $g$ on $\Sigma$; if $f: \Sigma \rightarrow \mathbb{R}$ is a Lipschitz function, we denote by $\nabla_{\tau} f$ the tangential gradient of $f$ on $\Sigma$.

Given a Lipschitz hypersurface $\Sigma \subset \mathbb{R}^{n}$ with boundary, we define the Minkowski content $\mathcal{M}_{\varphi}(\Sigma)$ of $\Sigma$ with respect to the norm $\varphi$ as

$$
\mathcal{M}_{\varphi}(\Sigma):=\liminf _{\rho \rightarrow 0^{+}} \frac{1}{2 \rho} \mathcal{H}^{n}\left(\left\{x \in \mathbb{R}^{n}: d_{\varphi}(x, \Sigma)<\rho\right\}\right)
$$

where $d_{\varphi}(x, \Sigma):=\inf \{\varphi(y-x): y \in \Sigma\}$. The quantity $\mathcal{M}_{\varphi}(\Sigma)$ is a surface measure naturally associated with $\varphi$ and $\Sigma$. We refer for instance to [7] for its use in geometric anisotropic evolution problems. It turns out that

$$
\mathcal{M}_{\varphi}(\Sigma)=\int_{\Sigma} \varphi^{o}(v) \mathrm{d} \mathcal{H}^{n-1}
$$

where $v(x)$ is a euclidean unit normal vector to $\Sigma$ at $\left(\mathcal{H}^{n-1}\right.$-almost every) $x \in \Sigma$. From the integral representation of $\mathcal{M}_{\varphi}(\Sigma)$ in $(5)$, it is natural to regard $\varphi^{o}(v)$ as the surface tension of a flat interface whose normal is $\nu$. We indicate by $\mathrm{d} \mathcal{P}_{\varphi}$ the measure on $\Sigma$ given by

$$
\mathrm{d} \mathcal{P}_{\varphi}(B):=\int_{B \cap \Sigma} \varphi^{o}(\nu) \mathrm{d} \mathcal{H}^{n-1}, \quad B \text { a Borel set. }
$$

At each point $x \in \mathbb{R}^{n}$ where $d_{\varphi}(x, \Sigma)$ is differentiable, we have $\nabla d_{\varphi}(x, \Sigma) \in \partial \mathcal{F}_{\varphi}$, that is,

$$
\varphi^{o}\left(\nabla d_{\varphi}(x, \Sigma)\right)=1
$$




\subsection{Partitions}

Given a locally finite family $\left\{E_{i}\right\}$ of open subsets of $\mathbb{R}^{n}$ with Lipschitz boundary such that $\bigcup_{i=1}^{\infty} \overline{E_{i}}=\mathbb{R}^{n}$ and $E_{i} \cap E_{j}=\emptyset$ for $i \neq j$, we say that $\left\{E_{i}\right\}$ is a Lipschitz (resp. smooth) partition of $\mathbb{R}^{n}$ if $\Sigma_{i j}:=\partial E_{i} \cap \partial E_{j}$ is a Lipschitz (resp. smooth) hypersurface with Lipschitz (resp. smooth) boundary.

For notational simplicity, when $n=2$ the sets $\partial E_{i} \cap \partial E_{j}$ are often denoted by $\Sigma_{k}$, using one index only.

Whenever $n=2$, by an $m$-multiple junction of $\left\{E_{i}\right\}$ ( $m \geqslant 3$ a natural number) we mean a point $q$ belonging to $m$ distinct arcs, where an arc is one of the $\Sigma_{i j}$. If $m=3$ we say that $q$ is a triple junction of $\left\{E_{i}\right\}$.

\section{First variation of $\mathcal{M}_{\varphi}$ in the smooth case}

Throughout all this section, we assume that $\varphi$ is smooth. Accordingly, we assume that $\Sigma$ is an $(n-1)$-dimensional smooth bounded embedded orientable manifold with (smooth) boundary. We recall that $v$ is a smooth euclidean unit normal vector field to $\Sigma$; we assume $v$ to be smoothly defined up to $\partial \Sigma$. At each point of $\bar{\Sigma}$ we define

- $v_{\varphi}:=v / \varphi^{o}(v)$;

- the $\varphi$-normal vector field $n_{\varphi}:=T^{o}\left(v_{\varphi}\right)=\varphi_{\xi}^{o}\left(v_{\varphi}\right)=\varphi_{\xi}^{o}(\nu)$, sometimes called the CahnHoffman field,

and at each point of $\Sigma$,

- the $\varphi$-mean curvature $\kappa_{\varphi}:=\operatorname{div}_{\tau} n_{\varphi}$ of $\Sigma$.

Concerning the previous definitions and their connections with geometric anisotropic evolution problems when $\partial \Sigma=\emptyset$ we refer for instance to [3].

DEFINITION 3.1 We denote by $n_{\varphi}^{\partial \Sigma}: \partial \Sigma \rightarrow \mathbb{R}^{n}$ the vector field defined as follows: if $x \in \partial \Sigma$ then

(i) $n_{\varphi}^{\partial \Sigma}(x) \in\left\{\operatorname{span}\left(T_{x}(\partial \Sigma), n_{\varphi}(x)\right)\right\}^{\perp}$;

(ii) $\left|n_{\varphi}^{\partial \Sigma}(x)\right|=\left|n_{\varphi}(x)-\Pi_{T_{x}(\partial \Sigma)} n_{\varphi}(x)\right|$;

(iii) $n_{\varphi}^{\partial \Sigma}(x)$ points outwards from $\Sigma$.

Observe that

$$
\operatorname{dim}\left\{\operatorname{span}\left(T_{x}(\partial \Sigma), n_{\varphi}(x)\right)^{\perp}\right\}=1 .
$$

This follows from the fact that $n_{\varphi}(x)$ and $T_{x}(\partial \Sigma)$ are linearly independent, which is a consequence of the property $n_{\varphi}(x) \cdot v_{\varphi}(x)=1$.

Note also that in $n=2$ dimensions condition (i) reduces to $n_{\varphi}^{\partial \Sigma}(x) \cdot n_{\varphi}(x)=0$, and condition (ii) reduces to $\left|n_{\varphi}^{\partial \Sigma}(x)\right|=\left|n_{\varphi}(x)\right|$.

REMARK 3.2 If $\varphi(\xi)=|\xi|$, then $n_{\varphi}^{\partial \Sigma}$ is the usual conormal unit euclidean vector pointing outwards from $\Sigma$.

REMARK 3.3 The vector field $n_{\varphi}^{\partial \Sigma}(x)$ is obtained by subtracting from $n_{\varphi}(x)$ its component in $T_{x}(\partial \Sigma)$, and then by performing a suitable rotation through $\pi / 2$ of the resulting vector (in the two-dimensional space $\left.T_{x}(\partial \Sigma)^{\perp}\right)$. 


\subsection{The smooth 2-dimensional case}

In this subsection we assume $n=2$ and we compute the first variation of $\mathcal{M}_{\varphi}$ using a parametric approach.

THEOREM 3.4 Let $\Sigma \subset \mathbb{R}^{2}$ be a smooth simple curve with boundary $\partial \Sigma=\{p, q\}$. Let $\gamma:[0,1] \rightarrow \mathbb{R}^{2}$ be a regular parametrization of $\Sigma$ with $\gamma(0)=p$ and $\gamma(1)=q$. Let $\beta \in \mathcal{C}^{2}\left([0,1] ; \mathbb{R}^{2}\right), \lambda \in \mathbb{R}$, and let $\Sigma_{\lambda}$ be the curve parametrized by $\gamma+\lambda \beta$. Then

$$
\frac{\mathrm{d}}{\mathrm{d} \lambda} \mathcal{M}_{\varphi}\left(\Sigma_{\lambda}\right)_{\mid \lambda=0}=\int_{\Sigma} \kappa_{\varphi} v_{\varphi} \cdot \beta \mathrm{d} \mathcal{P}_{\varphi}+n_{\varphi}^{\partial \Sigma}(q) \cdot \beta(1)+n_{\varphi}^{\partial \Sigma}(p) \cdot \beta(0) .
$$

Proof. Set $\tau:=\gamma^{\prime} /\left|\gamma^{\prime}\right|$ and $v:=\tau^{\perp}$. Recalling 5 we have

$$
\begin{aligned}
\frac{\mathrm{d}}{\mathrm{d} \lambda} \mathcal{M}_{\varphi}\left(\Sigma_{\lambda}\right)_{\mid \lambda=0} & =\frac{\mathrm{d}}{\mathrm{d} \lambda} \int_{0}^{1} \varphi^{o}\left(\left(\gamma^{\prime}+\lambda \beta^{\prime}\right)^{\perp}\right) \mathrm{d} t_{\mid \lambda=0} \\
& =\int_{0}^{1} \varphi_{\xi}^{o}(v) \cdot\left(\beta^{\perp}\right)^{\prime} \mathrm{d} t \\
& =-\int_{0}^{1} \frac{d}{d t}\left(\varphi_{\xi}^{o}(v)\right) \cdot \beta^{\perp} \mathrm{d} t-\varphi_{\xi}^{o}(v(q))^{\perp} \cdot \beta(1)+\varphi_{\xi}^{o}(v(p))^{\perp} \cdot \beta(0) .
\end{aligned}
$$

We now observe that $\beta^{\perp}=-\beta \cdot \nu \tau+\beta \cdot \tau \nu$. Moreover, $\varphi_{\xi}^{o}(\nu)=n_{\varphi}$ by definition, and from [6, Proposition 3.1, Example 4.2] we have $\varphi_{\xi \xi}^{o}(v) \tau \cdot v=0$ and $\kappa_{\varphi}=\kappa \varphi_{\xi \xi}^{o}(v) \tau \cdot \tau$, where $\kappa$ is the euclidean curvature. Therefore

$$
\int_{0}^{1} \frac{\mathrm{d}}{\mathrm{d} t}\left(\varphi_{\xi}^{o}(\nu)\right) \cdot \beta^{\perp} \mathrm{d} t=-\int_{0}^{1} \kappa \varphi_{\xi \xi}^{o}(\nu) \tau \cdot \tau \beta \cdot \nu\left|\gamma^{\prime}\right| \mathrm{d} t=-\int_{\Sigma} \kappa_{\varphi} v_{\varphi} \cdot \beta \mathrm{d} \mathcal{P}_{\varphi} .
$$

Then (9) follows from (10) and (11).

COROLlary 3.5 Let $\left\{E_{i}\right\}$ be a smooth partition of $\mathbb{R}^{2}$ and let $q$ be an $m$-multiple junction of $\left\{E_{i}\right\}$, $m \geqslant 3$. Let $\Sigma_{1}, \ldots, \Sigma_{m}$ be the $m$ arcs of the partition meeting at $q$, and set $T:=\bigcup_{i=1}^{m} \Sigma_{i}$. Let $\gamma_{i}:[0,1] \rightarrow \mathbb{R}^{2}$ be a regular parametrization of $\Sigma_{i}$ such that $\gamma_{i}(1)=q$ for $i=1, \ldots, m$. Let $\beta_{i} \in \mathcal{C}^{2}\left([0,1] ; \mathbb{R}^{2}\right)$ be such that $\beta_{i}(0)=0$ and $\beta_{i}(1)=\beta_{j}(1)=: \beta(1)$ for every $i, j \in\{1, \ldots, m\}$, let $\lambda \in \mathbb{R}$ and $\Sigma_{\lambda}^{i}$ be the curve parametrized by $\gamma_{i}+\lambda \beta_{i}$, and set $T_{\lambda}:=\bigcup_{i=1}^{m} \Sigma_{\lambda}^{i}$. Then

$$
\frac{\mathrm{d}}{\mathrm{d} \lambda} \mathcal{M}_{\varphi}\left(T_{\lambda}\right)_{\left.\right|_{\lambda=0}}=\int_{T} \kappa_{\varphi} v_{\varphi} \cdot \beta \mathrm{d} \mathcal{P}_{\varphi}+\beta(1) \cdot \sum_{i=1}^{m} n_{\varphi}^{\partial \Sigma_{i}}(q)
$$

In particular, if for any $\beta_{i}$ as above we have $\frac{\mathrm{d}}{\mathrm{d} \lambda} \mathcal{M}_{\varphi}\left(T_{\lambda}\right)_{\left.\right|_{\lambda=0}}=0$, then each $\Sigma_{i}$ has zero $\varphi$-mean curvature, and

$$
\sum_{i=1}^{m} n_{\varphi}^{\partial \Sigma_{i}}(q)=0
$$

We call condition (13) the balance condition at $q$. 


\subsection{The smooth $n$-dimensional case}

In this subsection we assume $n \geqslant 2$ and we compute the first variation of $\mathcal{M}_{\varphi}$. Given a $\mathcal{C}^{1}$ vector field $g: \mathbb{R}^{n} \rightarrow \mathbb{R}^{n}$ we set $\operatorname{div}_{\varphi, \tau} g:=\operatorname{tr}\left(\left(\mathrm{Id}-n_{\varphi} \otimes v_{\varphi}\right) \nabla g\right)$. The next result was proved, for $\partial \Sigma=\emptyset$, in [3].

THEOREM 3.6 Let $\Sigma \subset \mathbb{R}^{n}$ be a smooth hypersurface with boundary. For $\lambda \in \mathbb{R}$, let $\psi_{\lambda}$ : $\mathbb{R}^{n} \rightarrow \mathbb{R}^{n}$ be a family of diffeomorphisms such that $\psi_{0}=$ Id and $\psi_{\lambda}-$ Id has compact support in $\mathbb{R}^{n}$. Set $\Sigma_{\lambda}:=\psi_{\lambda}(\Sigma)$. Then

$$
\frac{d}{d \lambda} \mathcal{M}_{\varphi}\left(\Sigma_{\lambda}\right)_{\left.\right|_{\lambda=0}}=\int_{\Sigma} \operatorname{div}_{\varphi, \tau} g \mathrm{~d} \mathcal{P}_{\varphi}=\int_{\Sigma} \kappa_{\varphi} v_{\varphi} \cdot g \mathrm{~d} \mathcal{P}_{\varphi}+\int_{\partial \Sigma} n_{\varphi}^{\partial \Sigma} \cdot g \mathrm{~d} \mathcal{H}^{n-2}
$$

where $g:=\frac{\partial \psi_{\lambda}}{\partial \lambda} \mid \lambda=0$.

Proof. By the area formula it is well known that

$$
\mathrm{d} \mathcal{H}^{n-1}\left(\psi_{\lambda}(x)\right)=\mathrm{d} \mathcal{H}^{n-1}(x)+\lambda \operatorname{div}_{\tau} g(x) \mathrm{d} \mathcal{H}^{n-1}(x)+o(\lambda) .
$$

Denoting by $v_{\lambda}$ a smooth euclidean unit normal vector field on $\Sigma_{\lambda}$, we obtain

$$
\begin{aligned}
\frac{d}{d \lambda} \mathcal{M}_{\varphi}\left(\Sigma_{\lambda}\right)_{\mid \lambda=0} & =\int_{\Sigma} \frac{\mathrm{d}}{\mathrm{d} \lambda} \varphi^{o}\left(v_{\lambda}\left(\psi_{\lambda}\right)\right)_{\left.\right|_{\lambda=0}} \mathrm{~d} \mathcal{H}^{n-1}+\int_{\Sigma} \varphi^{o}(\nu) \operatorname{div}_{\tau} g \mathrm{~d} \mathcal{H}^{n-1} \\
& =\int_{\Sigma} n_{\varphi} \cdot \frac{\mathrm{d}}{\mathrm{d} \lambda} v_{\lambda}\left(\psi_{\lambda}\right)_{\left.\right|_{\lambda=0}} \mathrm{~d} \mathcal{H}^{n-1}+\int_{\Sigma} \varphi^{o}(\nu) \operatorname{div}_{\tau} g \mathrm{~d} \mathcal{H}^{n-1} .
\end{aligned}
$$

Following [6] one can prove that even if $g$ is not necessarily $\varphi$-normal, we have

$$
\frac{\mathrm{d}}{\mathrm{d} \lambda} \nu_{\lambda}\left(\psi_{\lambda}\right)_{\mid \lambda=0}=-v \nabla g+(\nu \cdot v \nabla g) v \quad \text { on } \Sigma .
$$

Hence

$$
\begin{aligned}
\frac{\mathrm{d}}{\mathrm{d} \lambda} \mathcal{M}_{\varphi}\left(\Sigma_{\lambda}\right)_{\mid \lambda=0} & =\int_{\Sigma} n_{\varphi} \cdot(-v \nabla g+(v \cdot v \nabla g) v) \mathrm{d} \mathcal{H}^{n-1}+\int_{\Sigma} \varphi^{o}(v) \operatorname{div}_{\tau} g \mathrm{~d} \mathcal{H}^{n-1} \\
& =\int_{\Sigma}\left(\operatorname{div} g-n_{\varphi} \cdot v_{\varphi} \nabla g\right) \mathrm{d} \mathcal{P}_{\varphi},
\end{aligned}
$$

which proves the first equality in (14).

For $\rho$ small enough let

$$
\begin{aligned}
U_{\rho} & :=\left\{x+\sigma n_{\varphi}(x): \sigma \in\right]-\rho, \rho[, x \in \Sigma\}, \\
\Sigma_{\rho}^{ \pm} & :=\left\{x \pm \rho n_{\varphi}(x): x \in \Sigma\right\}, \\
\Sigma_{\rho} & :=\left\{x+\sigma n_{\varphi}(x): x \in \partial \Sigma, \sigma \in\right]-\rho, \rho[\} .
\end{aligned}
$$

Let $g^{e}: U_{\rho} \rightarrow \mathbb{R}^{n}$ be defined as $g^{e}(y):=g\left(y-\bar{d}_{\varphi}(y, \Sigma) T^{o}\left(\nabla \bar{d}_{\varphi}(y, \Sigma)\right)\right)$, where $\bar{d}_{\varphi}(\cdot, \Sigma):=$ $d_{\varphi}(\cdot, \Sigma)$ on $\left\{x+\sigma n_{\varphi}(x): \sigma \in\left[0, \rho[, x \in \Sigma\}\right.\right.$ and $\bar{d}_{\varphi}(\cdot, \Sigma):=-d_{\varphi}(\cdot, \Sigma)$ on $\left\{x+\sigma n_{\varphi}(x): \sigma \in\right.$ ] $-\rho, 0[, x \in \Sigma$ ]. Using the coarea formula, (7), and the divergence theorem it is not difficult to check that

$$
\int_{\Sigma} \operatorname{div}_{\varphi, \tau} g \mathrm{~d} \mathcal{P}_{\varphi}=\lim _{\rho \rightarrow 0^{+}} \frac{1}{2 \rho} \int_{U_{\rho}} \operatorname{div}\left(g^{e}\right) \mathrm{d} x=\lim _{\rho \rightarrow 0^{+}}\left[I_{\rho}+I I_{\rho}\right],
$$


where

$$
I_{\rho}:=\frac{1}{2 \rho}\left[\int_{\Sigma_{\rho}^{+}} g^{e} \cdot \tilde{v}_{\rho}^{+} \mathrm{d} \mathcal{H}^{n-1}+\int_{\Sigma_{\rho}^{-}} g^{e} \cdot \widetilde{v}_{\rho}^{-} \mathrm{d} \mathcal{H}^{n-1}\right], \quad I I_{\rho}:=\frac{1}{2 \rho} \int_{\Sigma_{\rho}} g^{e} \cdot \widetilde{v}_{\rho} \mathrm{d} \mathcal{H}^{n-1},
$$

and $\widetilde{v}_{\rho}^{ \pm}$and $\widetilde{v}_{\rho}$ are the unit euclidean normal vectors respectively to $\Sigma_{\rho}^{ \pm}$and $\Sigma_{\rho}$, pointing outwards from $U_{\rho}$.

Using the area formula and applying 15 with $g(\cdot)=T^{o}\left(\nabla \bar{d}_{\varphi}(\cdot, \Sigma)\right)$ one can check that

$$
\lim _{\rho \rightarrow 0^{+}} I_{\rho}=\int_{\Sigma} \kappa_{\varphi} v_{\varphi} \cdot g \mathrm{~d} \mathcal{P}_{\varphi}
$$

Therefore, in view of (17), to conclude the proof of the last equality in (14) we have to show that

$$
\lim _{\rho \rightarrow 0^{+}} I I_{\rho}=\int_{\partial \Sigma} n_{\varphi}^{\partial \Sigma} \cdot g \mathrm{~d} \mathcal{H}^{n-2}
$$

Let $T \Sigma_{\rho}$ be the tangent bundle to $\Sigma_{\rho}$ and let $f_{\rho}: T \Sigma_{\rho} \rightarrow[0,+\infty[$ be the Finsler norm obtained as "restriction of $\varphi$ to $\Sigma_{\rho}$ ", defined as follows:

$$
\left\{\xi \in T_{x}\left(\Sigma_{\rho}\right): f_{\rho}(x, \xi) \leqslant 1\right\}=\mathcal{W}_{\varphi} \cap T_{x}\left(\Sigma_{\rho}\right), \quad x \in \Sigma_{\rho}
$$

For any $x, y \in \Sigma_{\rho}$, let

$$
d_{f_{\rho}}(x, y):=\inf \left\{\int_{0}^{1} \varphi(\gamma, \dot{\gamma}) \mathrm{d} t: \gamma \in A C\left([0,1] ; \mathbb{R}^{n}\right), \gamma(0)=x, \gamma(1)=y, \gamma(t) \in T_{\gamma(t)}\left(\Sigma_{\rho}\right)\right\},
$$

and let $d_{\rho}: \Sigma_{\rho} \rightarrow \mathbb{R}$ be defined as follows: $d_{\rho}(y):=d_{f_{\rho}}(y, \partial \Sigma)$ if $y=x+\sigma n_{\varphi}(x), x \in \partial \Sigma$ and $\sigma \geqslant 0$, and $d_{\rho}(y):=-d_{f_{\rho}}(y, \partial \Sigma)$ if $y=x+\sigma n_{\varphi}(x), x \in \partial \Sigma$ and $\sigma<0$.

Using the coarea formula on manifolds [12] and recalling that $\nabla_{\tau} d_{\rho}$ is the tangential gradient of $d_{\rho}$ on $\Sigma_{\rho}$, we have

$$
I I_{\rho}=\frac{1}{2 \rho} \int_{-\rho}^{\rho} d \sigma \int_{\left\{x \in \Sigma_{\rho}: d_{\rho}(x)=\sigma\right\}} \frac{g^{e} \cdot \widetilde{v}_{\rho}}{\left|\nabla_{\tau} d_{\rho}\right|} \mathrm{d} \mathcal{H}^{n-2} .
$$

Using the eikonal equation $f_{\rho}^{o}\left(x, \nabla_{\tau} d_{\rho}(x)\right)=1$ where $f_{\rho}^{o}\left(x, \xi^{o}\right):=\sup \left\{\xi \cdot \xi^{o}: f_{\rho}(x, \xi) \leqslant 1\right\}$ for any $\left(x, \xi^{o}\right)$ in the cotangent bundle of $\Sigma_{\rho}$, we have

$$
I I_{\rho}=\frac{1}{2 \rho} \int_{-\rho}^{\rho} d \sigma \int_{\left\{x \in \Sigma_{\rho}: d_{\rho}(x)=\sigma\right\}} g^{e}(x) \cdot \widetilde{v}_{\rho}(x) f_{\rho}^{o}\left(x, \frac{\nabla_{\tau} d_{\rho}(x)}{\left|\nabla_{\tau} \mathrm{d}_{\rho}(x)\right|}\right) d \mathcal{H}^{n-2}(x) .
$$

Letting $\rho \rightarrow 0^{+}$and setting

$$
V(x):=\operatorname{span}\left\{T_{x}(\partial \Sigma), n_{\varphi}(x)\right\}, \quad x \in \partial \Sigma,
$$

we get

$$
\lim _{\rho \rightarrow 0^{+}} I I_{\rho}=\int_{\partial \Sigma} g \cdot \widetilde{v} f_{0}^{o}(x, \eta(x)) \mathrm{d} \mathcal{H}^{n-2},
$$

where 
(a) $\widetilde{v}: \partial \Sigma \rightarrow \mathbb{R}^{n}$ is the vector field pointing outwards from $\Sigma$ determined by the following conditions:

$$
\widetilde{v}(x) \in V(x)^{\perp}, \quad|\widetilde{v}(x)|=1, \quad x \in \partial \Sigma ;
$$

(b) $\eta: \partial \Sigma \rightarrow \mathbb{R}^{n}$ is the vector field determined (up to sign) by the following conditions:

$$
\eta(x) \in T_{x}(\partial \Sigma)^{\perp} \cap V(x), \quad|\eta(x)|=1, \quad x \in \partial \Sigma ;
$$

(c) $f_{0}^{o}\left(x, \xi^{o}\right):=\sup \left\{\xi \cdot \xi^{o}: \xi \in \mathcal{W}_{\varphi} \cap V(x)\right\}, x \in \partial \Sigma$.

To conclude the proof of (18) it is sufficient to show that

$$
n_{\varphi}^{\partial \Sigma}(x)=f_{0}^{o}(x, \eta(x)) \widetilde{v}(x), \quad x \in \partial \Sigma .
$$

To this end we observe that, thanks to 8 , (i) of Definition 3.1 and (a), the vectors $\widetilde{v}(x)$ and $n_{\varphi}^{\partial \Sigma}(x)$ are parallel and point in the same direction. Moreover,

$$
\eta(x)= \pm \frac{\Pi_{V(x)} v_{\varphi}(x)}{\left|\Pi_{V(x)} v_{\varphi}(x)\right|} .
$$

Observe now that, by definition, the normal to $\mathcal{W}_{\varphi}$ at $n_{\varphi}(x)$ is $v_{\varphi}(x) /\left|v_{\varphi}(x)\right|$. Therefore the normal to $\mathcal{W}_{\varphi} \cap \partial V(x)$ at $n_{\varphi}(x)$ (in the space $V(x)$ ) is $\Pi_{V(x)} v_{\varphi}(x) /\left|\Pi_{V(x)} v_{\varphi}(x)\right|= \pm \eta(x)$. This implies that the supremum defining $f_{0}^{o}(x, \eta(x))$ (see (c)) is attained at $n_{\varphi}(x)$, i.e.,

$$
f_{0}^{o}(x, \eta(x))=\left|n_{\varphi}(x) \cdot \eta(x)\right| .
$$

Hence

$$
f_{0}^{o}(x, \eta(x))=\left|n_{\varphi}(x) \cdot \eta(x)\right|=\left|n_{\varphi}(x)-\Pi_{T_{x}(\partial \Sigma)} n_{\varphi}(x)\right|=\left|n_{\varphi}^{\partial \Sigma}(x)\right| .
$$

REMARK 3.7 In the case $\varphi(\xi)=|\xi|$, the above argument gives the classical divergence theorem on a manifold with boundary.

REMARK 3.8 If $n=2$, formula (14) reduces to 9).

Corollary 3.9 Let $\left\{E_{i}\right\}$ be a smooth partition of $\mathbb{R}^{n}$ and let $\Sigma_{i j}:=\partial E_{i} \cap \partial E_{j}, T:=\bigcup_{i, j} \Sigma_{i j}$, $\Gamma:=\bigcup_{i, j} \partial \Sigma_{i j}$. For $\lambda \in \mathbb{R}$, let $\psi_{\lambda}: \mathbb{R}^{n} \rightarrow \mathbb{R}^{n}$ be a family of diffeomorphisms such that $\psi_{0}=\mathrm{Id}$ and $\psi_{\lambda}-\operatorname{Id}$ has compact support in $\mathbb{R}^{n}$. Set $\Sigma_{\lambda}^{i j}:=\psi_{\lambda}\left(\Sigma_{i j}\right)$ and $T_{\lambda}:=\bigcup_{i=1}^{m} \Sigma_{\lambda}^{i j}$. Then

$$
\frac{\mathrm{d}}{\mathrm{d} \lambda} \mathcal{M}_{\varphi}\left(T_{\lambda}\right)_{\mid \lambda=0}=\int_{T} \kappa_{\varphi} v_{\varphi} \cdot g \mathrm{~d} \mathcal{P}_{\varphi}+\int_{\Gamma}\left(\sum_{i, j} n_{\varphi}^{\partial \Sigma_{i j}}\right) \cdot g \mathrm{~d} \mathcal{H}^{n-2},
$$

where $g:=\frac{\partial \psi_{\lambda}}{\partial \lambda} \mid \lambda=0$. In particular, if for any $\psi_{\lambda}$ as above we have $\frac{\mathrm{d}}{\mathrm{d} \lambda} \mathcal{M}_{\varphi}\left(T_{\lambda}\right)_{\left.\right|_{\lambda=0}}=0$, then each $\Sigma_{i j}$ has zero $\varphi$-mean curvature and

$$
\sum_{i, j} n_{\varphi}^{\partial \Sigma_{i j}}=0 \quad \text { on } \Gamma
$$

We call condition (24) the ( $n$-dimensional version of the) balance condition on $\Gamma$; this condition is, in three dimensions, locally equivalent to condition (21) of [19].

REMARK 3.10 Condition (24) is equivalent to requiring that for any $q \in \Gamma$ there exist an open neighbourhood $U$ of $q$ and constants $\delta_{i j} \in\{-1,1\}$ (possibly depending on $U$ ) such that $\sum_{i, j} \delta_{i j} n_{\varphi \mid \Sigma_{i j}}(p)=0$ for any $p \in \Gamma \cap U$. 


\section{First variation of $\mathcal{M}_{\varphi}$ in the crystalline case}

To state the main result (Theorem 4.8) we need some preliminaries. Let $\Sigma \subset \mathbb{R}^{n}$ be a Lipschitz hypersurface with boundary. In the following any Lipschitz function or vector field defined on $\Sigma$ will be considered as defined up to $\partial \Sigma$. We denote by $v$ an $\mathcal{H}^{n-1}$-almost everywhere defined euclidean unit normal to $\Sigma$ and we set, as usual, $v_{\varphi}:=\nu / \varphi^{o}(\nu)$. We denote by $\operatorname{Lip}\left(\Sigma ; \mathbb{R}^{n}\right)$ the set of all Lipschitz vector fields on $\Sigma$, and define

$$
\begin{aligned}
\operatorname{Nor}_{\varphi}\left(\Sigma ; \mathbb{R}^{n}\right) & :=\left\{X: \Sigma \rightarrow \mathbb{R}^{n}: X(x) \in T^{o}\left(v_{\varphi}(x)\right) \text { for } \mathcal{H}^{n-1} \text {-a.e. } x \in \Sigma\right\}, \\
\operatorname{Lip}_{v, \varphi}\left(\Sigma ; \mathbb{R}^{n}\right) & :=\operatorname{Lip}\left(\Sigma ; \mathbb{R}^{n}\right) \cap \operatorname{Nor}_{\varphi}\left(\Sigma ; \mathbb{R}^{n}\right), \\
L_{\tau}^{2}\left(\Sigma ; \mathbb{R}^{n}\right) & :=\left\{N \in L^{2}\left(\Sigma ; \mathbb{R}^{n}\right): N(x) \cdot v_{\varphi}(x)=0 \text { for } \mathcal{H}^{n-1} \text {-a.e. } x \in \Sigma\right\}, \\
\operatorname{Lip}_{\tau}\left(\Sigma ; \mathbb{R}^{n}\right) & :=\left\{X \in \operatorname{Lip}\left(\Sigma ; \mathbb{R}^{n}\right): X(x) \cdot v_{\varphi}(x)=0 \text { for } \mathcal{H}^{n-1} \text {-a.e. } x \in \Sigma\right\}, \\
\operatorname{Lip}_{c}(\Sigma) & :=\{\psi \in \operatorname{Lip}(\Sigma): \operatorname{spt}(\psi) \cap \partial \Sigma=\emptyset\} .
\end{aligned}
$$

The following definition is the same as in [3, Definition 2.1], where it was introduced in the case $\partial \Sigma=\emptyset$.

DEFINITION 4.1 Let $\Sigma \subset \mathbb{R}^{n}$ be a Lipschitz hypersurface with boundary. We say that $\Sigma$ is Lipschitz $\varphi$-regular if there exists a vector field $n_{\varphi} \in \operatorname{Lip}_{v, \varphi}\left(\Sigma ; \mathbb{R}^{n}\right)$. We denote by $\mathcal{R}_{\varphi}^{\partial}\left(\mathbb{R}^{n}\right)$ the class of all Lipschitz $\varphi$-regular hypersurfaces.

Even in the case $\partial \Sigma=\emptyset$, the geometry of Lipschitz $\varphi$-regular sets is nontrivial and strictly related to the geometry of $\mathcal{W}_{\varphi}$ (see [4, Section 4], [2, Figure 7]).

With a little abuse of notation, we sometimes write $\left(\Sigma, n_{\varphi}\right) \in \mathcal{R}_{\varphi}^{\partial}\left(\mathbb{R}^{n}\right)$, and we say that $\left(\Sigma, n_{\varphi}\right)$ is Lipschitz $\varphi$-regular.

We now define the $\varphi$-weak tangential divergence of a vector field; we follow the definition given in [3, Definition 4.1] for the case $\partial \Sigma=\emptyset$, the only difference being that the operator is now tested on compactly supported Lipschitz functions. We refer to [3] for the motivations of such a definition and for an explanation why it generalizes the definition of $\operatorname{div}_{\varphi, \tau}$ given in subsection 3.2

Definition 4.2 Let $\left(\Sigma, n_{\varphi}\right) \in \mathcal{R}_{\varphi}^{\partial}\left(\mathbb{R}^{n}\right)$ and let $v \in L^{2}\left(\Sigma ; \mathbb{R}^{n}\right)$. We define the function $\operatorname{div}_{\varphi, n_{\varphi}, \tau} v: \operatorname{Lip}_{c}(\Sigma) \rightarrow \mathbb{R}$ as follows: for any $\psi \in \operatorname{Lip}_{c}(\Sigma)$ we set

$$
\left\langle\operatorname{div}_{\varphi, n_{\varphi}, \tau} v, \psi\right\rangle:=\int_{\Sigma} \psi v \cdot v_{\varphi} \operatorname{div}_{\tau} n_{\varphi} \mathrm{d} \mathcal{P}_{\varphi}-\int_{\Sigma}\left[\nabla_{\tau} \psi-\left(n_{\varphi} \cdot \nabla_{\tau} \psi\right) v_{\varphi}\right] \cdot v \mathrm{~d} \mathcal{P}_{\varphi} .
$$

Let

$$
\begin{aligned}
& H_{\tau, \varphi}^{\mathrm{div}}\left(\Sigma ; \mathbb{R}^{n}\right):=\left\{N \in L_{\tau}^{2}\left(\Sigma ; \mathbb{R}^{n}\right): \operatorname{div}_{\varphi, n_{\varphi}, \tau} N \in L^{2}(\Sigma)\right\}, \\
& H_{\nu, \varphi}^{\mathrm{div}}\left(\Sigma ; \mathbb{R}^{n}\right):=\left\{N \in \operatorname{Nor}_{\varphi}\left(\Sigma ; \mathbb{R}^{n}\right): \operatorname{div}_{\varphi, n_{\varphi}, \tau} N \in L^{2}(\Sigma)\right\} .
\end{aligned}
$$

REMARK 4.3 For $v \in H_{\tau, \varphi}^{\mathrm{div}}\left(\Sigma ; \mathbb{R}^{n}\right) \cup H_{\nu, \varphi}^{\mathrm{div}}\left(\Sigma ; \mathbb{R}^{n}\right)$, the operator $\operatorname{div}_{\varphi, n_{\varphi}, \tau} v$ does not depend on the choice of $n_{\varphi}$ in $\operatorname{Lip}_{v, \varphi}\left(\Sigma ; \mathbb{R}^{n}\right)$ (see [3, (A2) of Lemma 4.4 and Corollary 4.7]). Accordingly we shall use the notation $\operatorname{div}_{\varphi, \tau} v$ in place of $\operatorname{div}_{\varphi, n_{\varphi}, \tau} v$. Moreover, if $\Sigma$ is contained in a hyperplane, the function $\operatorname{div}_{\varphi, \tau} v$ coincides with the usual (weak) tangential divergence $\operatorname{div}_{\tau} v$ (see [4, Remark 2.2]).

The following definition is suggested by Definition 3.1 . 
Definition 4.4 Let $x \in \partial \Sigma$ be such that both $T_{x}(\bar{\Sigma})$ and $T_{x}(\partial \Sigma)$ exist, and let $z \in \mathbb{R}^{n} \backslash T_{x}(\bar{\Sigma})$. We define the vector $z^{\partial \Sigma} \in T_{x}(\partial \Sigma)^{\perp}$ as the rotation through $\pi / 2$ of the vector $z-\Pi_{T_{x}(\partial \Sigma)} z$ in such a way that $z^{\partial \Sigma}$ points outwards from $\Sigma$.

Given an $\mathcal{H}^{n-2}$-almost everywhere defined vector field $N: \partial \Sigma \rightarrow \mathbb{R}^{n}$ which is nontangent to $\bar{\Sigma}$, we define $N^{\partial \Sigma}: \partial \Sigma \rightarrow \mathbb{R}^{n}$ as $N^{\partial \Sigma}(x):=(N(x))^{\partial \Sigma}$.

ASSUMPTION To simplify the computations, from now on we will assume that $\varphi$ is crystalline and that the partitions $T$ are polyhedral. See Remark 4.11 for a discussion on when such an assumption can be weakened.

PROPOSITION 4.5 Let $\left(\Sigma, n_{\varphi}\right) \in \mathcal{R}_{\varphi}^{\partial}\left(\mathbb{R}^{n}\right)$ and assume that $\Sigma$ polyhedral. For any vector field $N \in H_{v, \varphi}^{\operatorname{div}}\left(\Sigma ; \mathbb{R}^{n}\right)$, there exists a function $\widehat{N} \in L^{\infty}\left(\partial \Sigma ; \mathbb{R}^{n}\right)$ such that

$$
\widehat{N}(x) \in T_{x}(\partial \Sigma)^{\perp} \quad \text { for } \mathcal{H}^{n-2} \text {-a.e. } x \in \partial \Sigma,
$$

and

$\int_{\Sigma} \psi \operatorname{div}_{\varphi, \tau} N \mathrm{~d} \mathcal{P}_{\varphi}=\int_{\Sigma} \psi \operatorname{div}_{\tau} n_{\varphi} \mathrm{d} \mathcal{P}_{\varphi}-\int_{\Sigma} \nabla_{\tau} \psi \cdot\left(N-n_{\varphi}\right) \mathrm{d} \mathcal{P}_{\varphi}-\int_{\partial \Sigma} \psi \widehat{N}^{\partial \Sigma} \cdot n_{\varphi} \mathrm{d} \mathcal{H}^{n-2}$

for any $\psi \in \operatorname{Lip}(\Sigma)$.

Proof. Let us first assume that $\Sigma$ is contained in a hyperplane. Let us denote by $v$ a unit normal vector to $\Sigma$ and by $\widetilde{v}$ the unit normal vector to $\partial \Sigma$ in the hyperplane containing $\Sigma$ and pointing outwards from $\Sigma$. Let us consider the tangent vector field $N-n_{\varphi}$. By Remark 4.3 we have $\operatorname{div}_{\varphi, \tau}\left(N-n_{\varphi}\right)=\operatorname{div}_{\tau}\left(N-n_{\varphi}\right) \mathcal{H}^{n-1}$-almost everywhere on $\Sigma$. Using the results of [1] (see also [16, Lemma 9.2]) we see that $N-n_{\varphi}$ admits a trace along $\widetilde{v}$ on $\partial \Sigma$, which we denote by $\left[N-n_{\varphi}, \widetilde{v}\right]$, and $\left[N-n_{\varphi}, \widetilde{v}\right] \in L^{\infty}(\partial \Sigma)$. Moreover, recalling $[6$, we have

$$
\begin{aligned}
\int_{\Sigma} \psi \operatorname{div}_{\varphi, \tau}(N & \left.-n_{\varphi}\right) \mathrm{d} \mathcal{P}_{\varphi}=\varphi^{o}(v) \int_{\Sigma} \psi \operatorname{div}_{\tau}\left(N-n_{\varphi}\right) \mathrm{d} \mathcal{H}^{n-1} \\
& =\varphi^{o}(v)\left(-\int_{\Sigma} \nabla_{\tau} \psi \cdot\left(N-n_{\varphi}\right) \mathrm{d} \mathcal{H}^{n-1}+\int_{\partial \Sigma} \psi\left[N-n_{\varphi}, \widetilde{v}\right] \mathrm{d} \mathcal{H}^{n-2}\right)
\end{aligned}
$$

for any $\psi \in \operatorname{Lip}(\Sigma)$. Let us define

$$
\widehat{N}(x):=n_{\varphi}(x)-\Pi_{T_{x}(\partial \Sigma)} n_{\varphi}(x)+\left[N-n_{\varphi}, \widetilde{v}\right] \widetilde{v}(x) \text { for } \mathcal{H}^{n-2} \text {-a.e. } x \in \partial \Sigma .
$$

Note that $\widehat{N}$ is nontangent to $\bar{\Sigma}$ and $\widehat{N}^{\partial \Sigma} \cdot n_{\varphi}=-\left[N-n_{\varphi}, \widetilde{v}\right] \widetilde{v} \cdot n_{\varphi}^{\partial \Sigma}$. Therefore 27 follows from 28 by observing that $\varphi^{o}(\nu)=\widetilde{v} \cdot n_{\varphi}^{\partial \Sigma}$.

Assume now that $\Sigma$ is polyhedral. We can reduce to the case in which $\Sigma$ is the union of two sheets $\Sigma_{1}, \Sigma_{2}$ each lying in a hyperplane. Since $N$ has square integrable divergence, it is not difficult to check that $\left(\widehat{N_{\mid \Sigma_{1}}}\right)^{\partial \Sigma_{1}}=-\left(\widehat{N_{\mid \Sigma_{2}}}\right)^{\partial \Sigma_{2}} \mathcal{H}^{n-2}$-almost everywhere on $\bar{\Sigma}_{1} \cap \bar{\Sigma}_{2}$. Then 27 follows from the previous case.

REMARK 4.6 Let $\zeta \in H_{\tau, \varphi}^{\operatorname{div}}\left(\Sigma ; \mathbb{R}^{n}\right)$. Reasoning as in Proposition 4.5 we can show that

$$
\int_{\Sigma} \psi \operatorname{div}_{\varphi, \tau} \zeta \mathrm{d} \mathcal{P}_{\varphi}=-\int_{\Sigma} \nabla_{\tau} \psi \cdot \zeta \mathrm{d} \mathcal{P}_{\varphi}+\int_{\partial \Sigma} \psi \zeta \cdot n_{\varphi}^{\partial \Sigma} \mathrm{d} \mathcal{H}^{n-2} \quad \forall \psi \in \operatorname{Lip}(\Sigma) .
$$




\subsection{Lipschitz $\varphi$-regular polyhedral partitions: statement of the main result}

The following definition is suggested by the results in subsection 3.2. In two dimensions, it is essentially the same definition given by J. E. Taylor in [28].

DEFINITION 4.7 Let $\left\{E_{i}\right\}$ be a Lipschitz partition of $\mathbb{R}^{n}$. For any $i \neq j$ let $\Sigma_{i j}:=\partial E_{i} \cap \partial E_{j}$ and $\Gamma:=\bigcup_{i, j} \partial \Sigma_{i j}$. We say that $\left\{E_{i}\right\}$ is Lipschitz $\varphi$-regular, and we write $\left\{E_{i}\right\} \in \mathcal{R} \mathcal{P}_{\varphi}\left(\mathbb{R}^{n}\right)$, if, for any $i \neq j, \Sigma_{i j} \in \mathcal{R}_{\varphi}^{\partial}\left(\mathbb{R}^{n}\right)$ and there exist vector fields $n_{\varphi}^{i j} \in \operatorname{Lip}_{v, \varphi}\left(\Sigma_{i j} ; \mathbb{R}^{n}\right)$ satisfying

$$
\sum_{i, j}\left(n_{\varphi}^{i j}\right)^{\partial \Sigma_{i j}}=0 \quad \text { on } \Gamma
$$

Let $\left\{E_{i}\right\} \in \mathcal{R} \mathcal{P}_{\varphi}\left(\mathbb{R}^{n}\right)$ and set $T:=\bigcup_{i, j} \Sigma_{i j}$. We denote by $H_{\nu, \varphi}^{\operatorname{div}}\left(T ; \mathbb{R}^{n}\right)\left(\operatorname{resp} . \operatorname{Nor}_{\varphi}\left(T ; \mathbb{R}^{n}\right)\right)$ the space of all vector fields $N: T \rightarrow \mathbb{R}^{n}$ such that $N_{\mid \Sigma_{i j}} \in H_{v, \varphi}^{\operatorname{div}}\left(\Sigma_{i j} ; \mathbb{R}^{n}\right)$ (resp. $N_{\mid \Sigma_{i j}} \in$ $\left.\operatorname{Nor}_{\varphi}\left(\Sigma_{i j} ; \mathbb{R}^{n}\right)\right)$ for any $i \neq j$.

Let $\left(\Sigma, n_{\varphi}\right) \in \mathcal{R}_{\varphi}^{\partial}\left(\mathbb{R}^{n}\right), \Sigma$ polyhedral, and $v \in \operatorname{Lip}\left(\Sigma ; \mathbb{R}^{n}\right)$. Since $\Sigma$ has a boundary, when computing the first variation of $\mathcal{M}_{\varphi}$ we cannot restrict ourselves to $\varphi$-normal vector fields, and tangent vector fields must be considered; as already remarked in the Introduction, this is one of the main additional difficulties in the computation of the first variation of $\mathcal{M}_{\varphi}$, as compared with [3].

Let $\left(\Sigma, n_{\varphi}\right) \in \mathcal{R}_{\varphi}^{\partial}\left(\mathbb{R}^{n}\right)$. Reasoning as in [3, Lemma 3.3], one finds that there exists $\rho>0$ such that the map $F_{n_{\varphi}}(x, t):=x+t n_{\varphi}(x)$, mapping $\left.\Sigma \times\right]-\rho, \rho$ [ onto its image, is bi-Lipschitz. We set $\left.F_{n_{\varphi}}^{-1}(\cdot)=\left(\pi_{n_{\varphi}}(\cdot), t_{n_{\varphi}}(\cdot)\right) \in \Sigma \times\right]-\rho, \rho[$.

For $t \in \mathbb{R}$ with $|t|<\rho, \rho>0$ small enough, define $U_{\rho}, \Sigma_{\rho}^{ \pm}$and $\Sigma_{\rho}$ as in 16 . Given a Lipschitz function $\psi$ and a Lipschitz vector field $\eta$ defined on $\Sigma$, we denote by $\psi^{e}:=\psi\left(\pi_{n_{\varphi}}\right): U_{\rho} \rightarrow \mathbb{R}$, $\eta^{e}:=\eta\left(\pi_{n_{\varphi}}\right): U_{\rho} \rightarrow \mathbb{R}^{n}$ the (Lipschitz) extensions of $\psi$ and $\eta$ respectively on $U_{\rho}$ along the vector field $n_{\varphi}$. Define $\widetilde{F}(z, t):=z+t v^{e}(z)$ on $U_{\rho}$. Set also $\widetilde{F}^{t}(\cdot):=\widetilde{F}(\cdot, t)$ and $\Sigma_{t}^{v}:=\widetilde{F}^{t}(\Sigma)$. Finally, let

$$
\operatorname{Var}\left(\mathcal{M}_{\varphi}, \Sigma\right)(v):=\liminf _{t \rightarrow 0^{+}} \frac{\mathcal{M}_{\varphi}\left(\Sigma_{t}^{v}\right)-\mathcal{M}_{\varphi}(\Sigma)}{t}
$$

Before proceeding with the computation, we split the vector field $v$ into its normal and tangential part as follows:

$$
v=\psi_{v} n_{\varphi}+t_{v}, \quad \psi_{v}:=v \cdot v_{\varphi}
$$

It is immediate to check that $t_{v} \cdot v_{\varphi}=0$, and therefore $t_{v}$ is tangent to $\Sigma$. We also set $\psi_{v}^{e}:=\left(\psi_{v}\right)^{e}$, $t_{v}^{e}:=\left(t_{v}\right)^{e}$, and

$$
\begin{aligned}
B_{\varphi}(\Sigma) & :=\left\{v \in \operatorname{Lip}\left(\Sigma ; \mathbb{R}^{n}\right): \psi_{v} \in \operatorname{Lip}(\Sigma), \int_{\Sigma}\left(\psi_{v}\right)^{2} \mathrm{~d} \mathcal{P}_{\varphi} \leqslant 1\right\}, \\
B_{\varphi}(T) & :=\left\{v \in \operatorname{Lip}\left(T ; \mathbb{R}^{n}\right): \psi_{v \mid \Sigma_{i j}} \in \operatorname{Lip}\left(\Sigma_{i j}\right) \forall i \neq j, \int_{T}\left(\psi_{v}\right)^{2} \mathrm{~d} \mathcal{P}_{\varphi} \leqslant 1\right\} .
\end{aligned}
$$

The vector field $\left(\int_{\Sigma}\left(n_{\varphi}\right)^{2} \mathrm{~d} \mathcal{P}_{\varphi}\right)^{-1} n_{\varphi}$ belongs to $B_{\varphi}(\Sigma)$. In particular, $B_{\varphi}(\Sigma)$ is nonempty. Notice also that if $v \in B_{\varphi}(\Sigma)$ then $t_{v}$ is a Lipschitz field. Finally, also $B_{\varphi}(T)$ is nonempty.

The main result of the paper is the following. 
THEOREM 4.8 Let $\varphi$ be crystalline. Let $\left\{E_{i}\right\} \in \mathcal{R} \mathcal{P}_{\varphi}\left(\mathbb{R}^{n}\right)$ be a polyhedral partition and let $\Gamma:=$ $\bigcup_{i, j} \partial \Sigma_{i j}$. Then

$$
\begin{aligned}
\inf _{v \in B_{\varphi}(T)} & \operatorname{Var}\left(\mathcal{M}_{\varphi}, T\right)(v) \\
\quad= & -\min \left\{\left[\int_{T}\left(\operatorname{div}_{\varphi, \tau} N\right)^{2} \mathrm{~d} \mathcal{P}_{\varphi}\right]^{1 / 2}: N \in H_{v, \varphi}^{\mathrm{div}}\left(T ; \mathbb{R}^{n}\right), \sum_{i, j}{\widehat{N_{\mid \Sigma_{i j}}}} \partial \Sigma_{i j}=0 \text { on } \Gamma\right\} .
\end{aligned}
$$

All examples in Section 5 are focused on explicit computation of a solution of the minimum problem on the right hand side of (34).

REMARK 4.9 Formula (34) gives, loosely speaking, the expression of (minus) the norm of the gradient of the functional $\mathcal{M}_{\varphi}$. If $N_{\min }$ is one minimizer of (34), then the (uniquely defined) quantity $\operatorname{div}_{\varphi, \tau} N_{\min }$ is expected to identify the initial velocity of $T$, if we consider $T$ as the initial datum for the crystalline mean curvature flow for partitions.

\subsection{Proof of Theorem 4.8}

Let us denote by $\Sigma$ one of the $\Sigma_{i j}$ of the partition. The proof of Theorem 4.8 is divided into five steps.

Step 1. We have

$$
\inf _{v \in B_{\varphi}(\Sigma)} \operatorname{Var}\left(\mathcal{M}_{\varphi}, \Sigma\right)(v)=\sup _{N \in H_{v, \varphi}^{\text {div }}\left(\Sigma ; \mathbb{R}^{n}\right)} \inf _{v \in B_{\varphi}(\Sigma)}\{I(N, v)+I I(N, v)\},
$$

where

$$
\begin{aligned}
I(N, v) & =\int_{\Sigma}\left(\psi_{v} \operatorname{div}_{\tau} n_{\varphi}-\nabla_{\tau} \psi_{v} \cdot\left(N-n_{\varphi}\right)\right) \mathrm{d} \mathcal{P}_{\varphi}, \\
I I(N, v) & =\int_{\Sigma}\left(-N \cdot v_{\varphi} \nabla t_{v}^{e}+\operatorname{div} t_{v}^{e}\right) \mathrm{d} \mathcal{P}_{\varphi} .
\end{aligned}
$$

Let $v \in B_{\varphi}(\Sigma)$. Following the same computations as in the proof of Theorem 5.1 in [3], it turns out that 32 can be written as

$$
\operatorname{Var}\left(\mathcal{M}_{\varphi}, \Sigma\right)(v)=\sup _{N \in \operatorname{Nor}_{\varphi}\left(\Sigma ; \mathbb{R}^{n}\right)}\{I(N, v)+I I(N, v)\},
$$

where $I(N, v)$ is as in $(36)$ and

$$
I I(N, v)=\int_{\Sigma}\left(-N \cdot v_{\varphi} \nabla t_{v}^{e}+v \cdot v \nabla t_{v}^{e}+\operatorname{div}_{\tau} t_{v}\right) \mathrm{d} \mathcal{P}_{\varphi} .
$$

Recalling the definition of the (euclidean) tangential divergence $\operatorname{div}_{\tau}$, we can rewrite $I I(N, v)$ as in (36). Using [37) and arguing as in [3, Proposition 5.2], we get

$$
\inf _{v \in B_{\varphi}(\Sigma)} \operatorname{Var}\left(\mathcal{M}_{\varphi}, \Sigma\right)(v)=\sup _{N \in \operatorname{Nor}_{\varphi}\left(\Sigma ; \mathbb{R}^{n}\right)} \inf _{v \in B_{\varphi}(\Sigma)}\{I(N, v)+I I(N, v)\} .
$$


Taking $v$ of the form $\psi n_{\varphi}, \psi \in \operatorname{Lip}_{c}(\Sigma)$, we have $I I(N, v)=0$ and $I(N, v)=\left\langle\operatorname{div}_{\varphi, \tau} N, \psi\right\rangle$. Hence $\inf _{B_{\varphi}(\Sigma)} I(N, v)=\inf _{B_{\varphi}(\Sigma)}\{I(N, v)+I I(N, v)\}=-\infty$ if $N \notin H_{v, \varphi}^{\mathrm{div}}\left(\Sigma ; \mathbb{R}^{n}\right)$, and 35$\}$ follows.

Step 2. The integral $I I(N, v)$ in 36 has the expression

$$
I I(N, v)=\int_{\partial \Sigma} v \cdot n_{\varphi}^{\partial \Sigma} \mathrm{d} \mathcal{H}^{n-2}
$$

and in particular it is independent of $N$.

Let $N \in H_{v, \varphi}^{\text {div }}\left(\Sigma ; \mathbb{R}^{n}\right)$. Since $t_{v}^{e}$ is Lipschitz continuous and tangential, we have $v_{\varphi} \nabla t_{v}^{e}=0$ $\mathcal{H}^{n-1}$-almost everywhere on $\Sigma$, hence from Remark 4.3 we get

$$
\operatorname{div}_{\varphi, \tau} t_{v}=\operatorname{div}_{\tau} t_{v}=\operatorname{div} t_{v}^{e}-N \cdot v_{\varphi} \nabla t_{v}^{e} \quad \mathcal{H}^{n-1} \text {-a.e. on } \Sigma .
$$

Therefore, from (41), 36) and (30) (applied with $\psi \equiv 1$ ) we obtain

$$
I I(N, v)=\int_{\Sigma} \operatorname{div}_{\varphi, \tau} t_{v} \mathrm{~d} \mathcal{P}_{\varphi}=\int_{\partial \Sigma} t_{v} \cdot n_{\varphi}^{\partial \Sigma} \mathrm{d} \mathcal{H}^{n-2}=\int_{\partial \Sigma} v \cdot n_{\varphi}^{\partial \Sigma} \mathrm{d} \mathcal{H}^{n-2},
$$

where the last equality follows from the decomposition 33 .

Step 3. We have

$$
\begin{aligned}
\inf _{v \in B_{\varphi}(\Sigma)} \operatorname{Var}\left(\mathcal{M}_{\varphi}, \Sigma\right)(v) & \\
\quad=\sup _{N \in H_{\nu, \varphi}^{\text {div }}\left(\Sigma ; \mathbb{R}^{n}\right)} & \inf _{v \in B_{\varphi}(\Sigma)}\left\{\int_{\Sigma} \psi_{v} \operatorname{div}_{\varphi, \tau} N \mathrm{~d} \mathcal{P}_{\varphi}+\int_{\partial \Sigma} v \cdot \widehat{N}^{\partial \Sigma} \mathrm{d} \mathcal{H}^{n-2}\right\} .
\end{aligned}
$$

If $N \in H_{v, \varphi}^{\operatorname{div}}\left(\Sigma ; \mathbb{R}^{n}\right)$, from 36 and 27 we obtain

$$
\begin{aligned}
I(N, v) & =\int_{\Sigma} \psi_{v} \operatorname{div}_{\varphi, \tau} N \mathrm{~d} \mathcal{P}_{\varphi}+\int_{\partial \Sigma} \psi_{v} \widehat{N}^{\partial \Sigma} \cdot n_{\varphi} \mathrm{d} \mathcal{H}^{n-2} \\
& =\int_{\Sigma} \psi_{v} \operatorname{div}_{\varphi, \tau} N \mathrm{~d} \mathcal{P}_{\varphi}+\int_{\partial \Sigma} \psi_{v}\left(\widehat{N}^{\partial \Sigma}-n_{\varphi}^{\partial \Sigma}\right) \cdot n_{\varphi} \mathrm{d} \mathcal{H}^{n-2}
\end{aligned}
$$

Taking into account 40, and (43) we get

$$
I(N, v)+I I(N, v)=\int_{\Sigma} \psi_{v} \operatorname{div}_{\varphi, \tau} N \mathrm{~d} \mathcal{P}_{\varphi}+\int_{\partial \Sigma}\left(v \cdot n_{\varphi}^{\partial \Sigma}+\psi_{v} n_{\varphi} \cdot\left(\widehat{N}^{\partial \Sigma}-n_{\varphi}^{\partial \Sigma}\right)\right) \mathrm{d} \mathcal{H}^{n-2}
$$

Since $\widehat{N}^{\partial \Sigma}-n_{\varphi}^{\partial \Sigma}$ is parallel to $v_{\varphi}$, recalling 33 we have

$$
\psi_{v} n_{\varphi} \cdot\left(\widehat{N}^{\partial \Sigma}-n_{\varphi}^{\partial \Sigma}\right)=v \cdot\left(\widehat{N}^{\partial \Sigma}-n_{\varphi}^{\partial \Sigma}\right)
$$

Hence (44) becomes

$$
I(N, v)+I I(N, v)=\int_{\Sigma} \psi_{v} \operatorname{div}_{\varphi, \tau} N \mathrm{~d} \mathcal{P}_{\varphi}+\int_{\partial \Sigma} v \cdot \widehat{N}^{\partial \Sigma} \mathrm{d} \mathcal{H}^{n-2},
$$

which, taking into account (39), gives 42. 
Step 4. Relation (34) holds with the infimum on the right hand side in place of the minimum.

Recalling that $T=\bigcup_{i, j} \Sigma_{i j}$, the definitions of $\operatorname{Nor}_{\varphi}\left(T ; \mathbb{R}^{n}\right)$ and of $H_{\nu, \varphi}^{\mathrm{div}}\left(T ; \mathbb{R}^{n}\right)$, when computing $\operatorname{Var}\left(\mathcal{M}_{\varphi}, T\right)(v)$ we can add the contributions of all $\Sigma_{i j}$. To simplify notation, we write $N_{i j}=\widehat{N}_{\mid \Sigma_{i j}} \partial \Sigma_{i j}$. Using step 3, we get,

$\inf _{v \in B_{\varphi}(T)} \operatorname{Var}\left(\mathcal{M}_{\varphi}, T\right)(v)=\sup _{N \in H_{v, \varphi}^{\text {div }}\left(T ; \mathbb{R}^{n}\right)} \inf _{v \in B_{\varphi}(T)}\left\{\int_{T} \psi_{v} \operatorname{div}_{\varphi, \tau} N \mathrm{~d} \mathcal{P}_{\varphi}+\int_{\Gamma} v \cdot\left(\sum_{i, j} N_{i j}\right) \mathrm{d} \mathcal{H}^{n-2}\right\}$.

Observe now that if for a vector field $N \in H_{v, \varphi}^{\text {div }}\left(T ; \mathbb{R}^{n}\right)$ we have $\sum_{i, j} N_{i j} \neq 0$ on $\Gamma$ then

$$
\inf _{v \in B_{\varphi}(T)}\left\{\int_{T} \psi_{v} \operatorname{div}_{\varphi, \tau} N \mathrm{~d} \mathcal{P}_{\varphi}+\int_{\Gamma} v \cdot\left(\sum_{i, j} N_{i j}\right) \mathrm{d} \mathcal{H}^{n-2}\right\}=-\infty .
$$

This follows from the fact that $T$ is polyhedral, hence we can arbitrarily fix $v$ on $\Gamma$ without violating the constraint $v \in B_{\varphi}(T)$ (in particular the fact that $\psi_{v} \in \operatorname{Lip}\left(T ; \mathbb{R}^{n}\right)$ ).

We finally obtain

$$
\begin{aligned}
\inf _{v \in B_{\varphi}(T)} \operatorname{Var}\left(\mathcal{M}_{\varphi}, T\right)(v) & =\sup _{N \in H_{v, \varphi}^{\operatorname{div}}\left(T ; \mathbb{R}^{n}\right), \sum_{i, j} N_{i j}=0} \inf _{v \in B_{\varphi}(T)}\left\{\int_{T} \psi_{v} \operatorname{div} \operatorname{div}_{\varphi, \tau} N \mathrm{~d} \mathcal{P}_{\varphi}\right\} \\
& =-\inf _{N \in H_{\nu, \varphi}^{\operatorname{div}}\left(T ; \mathbb{R}^{n}\right), \sum_{i, j} N_{i j}=0}\left[\int_{T}\left(\operatorname{div}_{\tau} N\right)^{2} \mathrm{~d} \mathcal{P}_{\varphi}\right]^{1 / 2} .
\end{aligned}
$$

Step 5. The minimum problem in 34 admits a solution $N_{\min } \in H_{v, \varphi}^{\operatorname{div}}\left(T ; \mathbb{R}^{n}\right)$. Moreover, if $N_{1}, N_{2}$ are two minimizers of 34 , then $\operatorname{div}_{\varphi, \tau} N_{1}=\operatorname{div}_{\varphi, \tau} N_{2} \mathcal{H}^{n-1}$-almost everywhere on $T$.

The set

$$
C:=\left\{\operatorname{div}_{\varphi, \tau} N: N \in H_{v, \varphi}^{\operatorname{div}}\left(T ; \mathbb{R}^{n}\right), \sum_{i, j} N_{i j}=0 \text { on } \Gamma\right\}
$$

is a closed convex subset of $L^{2}(T)$. Indeed, the convexity follows from the fact that $H_{\nu, \varphi}^{\text {div }}\left(T ; \mathbb{R}^{n}\right)$ is a convex subset of the Hilbert space $\left\{N \in L^{2}\left(T ; \mathbb{R}^{n}\right): \operatorname{div}_{\varphi, \tau} N \in L^{2}(T)\right\}$, and the condition $\sum_{i, j} N_{i j}=0$ is linear.

Following [3], let us prove that $C$ is closed. Let $f_{k}:=\operatorname{div}_{\varphi, \tau} N_{k} \in C$ be such that $f_{k} \rightarrow f$ in $L^{2}(T)$ as $k \rightarrow \infty$. We have to prove that $f \in C$. Since $\sup _{k}\left\|N_{k}\right\|_{L^{2}\left(T ; \mathbb{R}^{n}\right)}<\infty$, possibly passing to a subsequence we can assume that $\left\{N_{k}\right\}$ converges weakly in $L^{2}\left(T ; \mathbb{R}^{n}\right)$ to a vector field $N \in L^{2}\left(T ; \mathbb{R}^{n}\right)$. Since $N_{k} \in \operatorname{Nor}_{\varphi}\left(T ; \mathbb{R}^{n}\right)$, we deduce that $N \in \operatorname{Nor}_{\varphi}\left(T ; \mathbb{R}^{n}\right)$. Moreover, for any $\psi \in \operatorname{Lip}(T)$ from 27$)$ we obtain

$$
\begin{aligned}
\int_{T} \psi f \mathrm{~d} \mathcal{P}_{\varphi} & =\lim _{k \rightarrow \infty} \int_{T} \psi \operatorname{div}_{\varphi, \tau} N_{k} \mathrm{~d} \mathcal{P}_{\varphi}=\int_{T} \psi \operatorname{div}_{\tau} n_{\varphi} \mathrm{d} \mathcal{P}_{\varphi}-\lim _{k \rightarrow \infty} \int_{T} \nabla_{\tau} \psi \cdot\left(N_{k}-n_{\varphi}\right) \mathrm{d} \mathcal{P}_{\varphi} \\
& =\int_{T} \psi \operatorname{div}_{\tau} n_{\varphi} \mathrm{d} \mathcal{P}_{\varphi}-\int_{T} \nabla_{\tau} \psi \cdot\left(N-n_{\varphi}\right) \mathrm{d} \mathcal{P}_{\varphi} .
\end{aligned}
$$

It follows that $f=\operatorname{div}_{\varphi, \tau} N$, hence $N \in H_{v, \varphi}^{\operatorname{div}}\left(T ; \mathbb{R}^{n}\right)$ and $\sum_{i, j} N_{i j}=0$ on $\Gamma$. Therefore $C$ is closed in $L^{2}\left(T ; \mathbb{R}^{n}\right)$. The conclusion now follows since the functional in 34 is strictly convex in the divergence.

The proof of the theorem is complete. 
REMARK 4.10 If $n=2$ the vector field $N_{\text {min }}$ is unique, since any vector field $N \in H_{v, \varphi}^{\mathrm{div}}\left(T ; \mathbb{R}^{2}\right)$ is uniquely determined by its divergence and the value at one point.

REMARK 4.11 If $n=2$ then $\operatorname{Lip}_{v, \varphi}\left(\Sigma ; \mathbb{R}^{2}\right)$ is dense in $H_{v, \varphi}^{\text {div }}\left(\Sigma ; \mathbb{R}^{2}\right)$. Indeed, any vector field $\xi \in$ $H_{v, \varphi}^{\text {div }}\left(\Sigma ; \mathbb{R}^{2}\right)$ is nonconstant only where $\Sigma$ coincides with a segment parallel to an edge of $\mathcal{W}_{\varphi}:$ on such segments the normal component of $\xi$ is constant and the tangential component belongs to $W^{1,2}$. Hence $\xi$ is continuous and can be approximated in $W^{1,2}$ by a sequence of Lipschitz continuous vector fields.

Finally, if $n=2$, Theorem 4.8 holds also when $T$ is piecewise $C^{1,1}$, instead of polygonal. Indeed, one can directly prove 27p under the assumption $N \in \operatorname{Lip}_{v, \varphi}\left(\Sigma ; \mathbb{R}^{2}\right)$, and then conclude by approximation and using the previous observation.

REMARK 4.12 All results of Sections 3 and 4 can be easily generalized in the presence of a family of Finsler norms $\varphi_{i j}$ (satisfying some compatibility conditions, see for instance [22]). For example, in the crystalline case, it is enough to consider the appropriate norm $\varphi_{i j}$ on each polyhedral interface dividing $E_{i}$ and $E_{j}$ and consequently to define the Cahn-Hoffman field $n_{\varphi_{i j}}$. In a similar way, it is possible to generalize relation (34) in the case of several norms.

\section{Examples}

In this section we show with some examples how formula (34) leads to an explicit computation of the crystalline mean curvature. As already observed in Remark 4.10, in two dimensions each edge of the partition has constant $\varphi$-curvature and the solution $N_{\min }$ of the minimum problem on the right hand side of (34) is unique; in three dimensions in general it is not true that each facet of the partition has constant $\varphi$-mean curvature already in the two-phases cases (see [2]). Moreover, uniqueness of $N_{\text {min }}$ in general is not expected; however, two solutions of 34 have the same divergence.

Recall that when $n=2$ the sets $\partial E_{i} \cap \partial E_{j}$ are often denoted by $\Sigma_{k}$; in this case $L_{k}$ denotes the length of $\Sigma_{k}$.

In two dimensions, we also give the following definition, whose meaning will be discussed at length in what follows.

DEFINITION 5.1 Let $\left\{E_{i}\right\}$ be a Lipschitz $\varphi$-regular partition of $\mathbb{R}^{2}$ and let $q$ be any multiple junction of $T$. Let $N_{\min }$ be the solution of (34). We say that $T$ is stable if, denoting by $\Sigma_{1}, \ldots, \Sigma_{m}$ all the edges of $T$ having $q$ as an extremum $(m \geqslant 3),\left(N_{\min }\right)_{\mid \Sigma_{i}}(q)$ is not a vertex of $\mathcal{W}_{\varphi}$ for any $i=1, \ldots, m$. We say that $T$ is unstable if it is not stable.

\subsection{Two-dimensional examples}

We begin with the two-dimensional case, where we assume that $\mathcal{W}_{\varphi}$ is a regular octagon centered at the origin (see Figure 1). We denote by $l$ its side length and by $r$ the radius of the circumscribed circle. The vectors $n_{i}^{a}$ and $n_{i}^{b}$ satisfy the balance condition $\sum_{i=1}^{3} n_{i}^{a}=\sum_{i=1}^{3} n_{i}^{b}=0$.

As shown by J. Taylor in [28], there are only eight possible configurations $T$ with one triple junction and $T \in \mathcal{R} \mathcal{P}_{\varphi}\left(\mathbb{R}^{2}\right)$ (see Figure 2); each of the three edges $\Sigma_{1}, \Sigma_{2}, \Sigma_{3}$ meeting at $q$ is parallel to an edge of $\mathcal{W}_{\varphi}$ and the possible configurations are given by the one in Figure 2 and by its rotations through multiples of $\pi / 4$.

Each of these configurations (assuming $q$ is not adjacent to another triple junction) gives rise to a different vector field $N_{\min }: T \rightarrow \mathbb{R}^{2}$ (the minimizing solution of 34 ). 


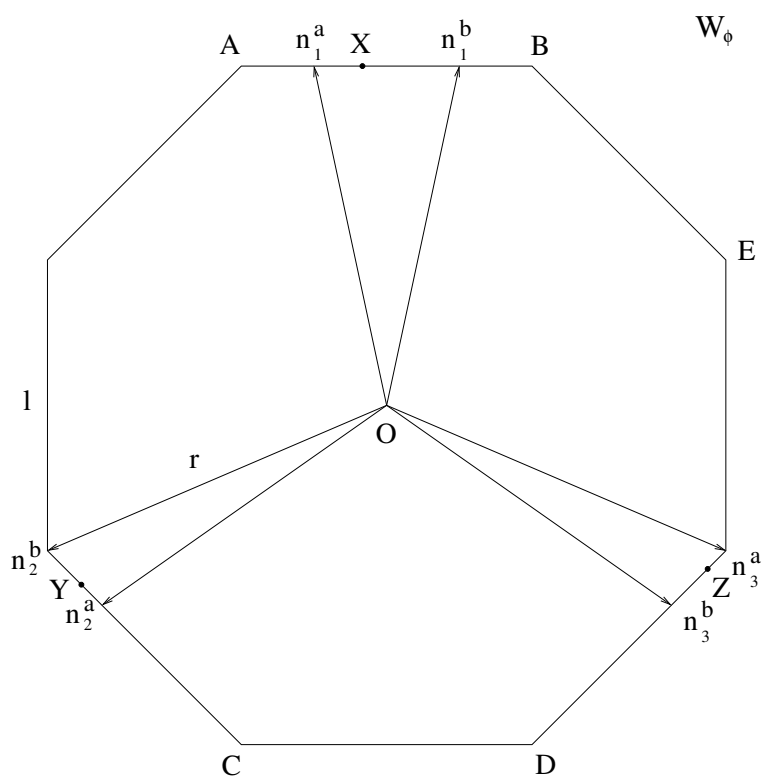

FIG. 1. The Wulff shape $\mathcal{W}_{\varphi}$. The vectors $n_{i}^{a}$ and $n_{i}^{b}\left(\sum_{i=1}^{3} n_{i}^{a}=\sum_{i=1}^{3} n_{i}^{b}=0\right)$ delimitate the admissible ranges of a field $N$ at a triple junction $q$. For instance, the vectors $X, Y, Z$ form an admissible triplet, i.e. $X+Y+Z=0$.

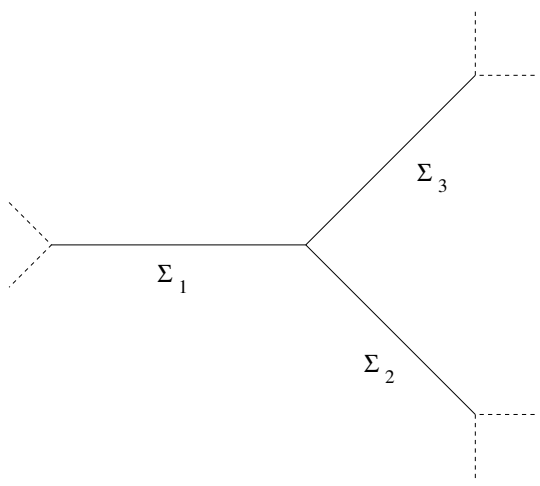

FIG. 2. Example of a Lipschitz $\varphi$-regular partition $T$ with one triple junction, when the Wulff shape is an octagon.

The balance condition

$$
\sum_{i, j}{\widehat{N_{\mid \Sigma_{i j}}}}^{\partial \Sigma_{i j}}=0 \quad \text { on } \Gamma
$$

appearing in 34 now reads

$$
N_{\mid \Sigma_{1}}(q)+N_{\mid \Sigma_{2}}(q)+N_{\mid \Sigma_{3}}(q)=0 .
$$

As shown in Figure 1, in order to satisfy the balance condition $(48)$ the vector $\left(N_{\min }\right)_{\mid \Sigma_{i}}(q)$ can take values only in the range between $n_{i}^{a}$ and $n_{i}^{b}, i=1,2,3$. 
Let us consider for instance the configuration $T$ in Figure 3 We want to explicitly compute the crystalline $\varphi$-curvature of $T$, at least in the special cases $L_{1}=L_{2}=L_{3}$ (Example 1) and $L_{1} \gg 1$ and $L_{2}=L_{3}$ (Example 2).

Note that $r:=\left|n_{2}^{b}\right|=l /(2 \sin (\pi / 8))$. Moreover, by symmetry we obtain

$$
\left|A-n_{1}^{a}\right|=\left|B-n_{1}^{b}\right|=\left|n_{3}^{a}-n_{3}^{b}\right|=\left|n_{2}^{a}-n_{2}^{b}\right|=: \delta .
$$

The balance condition $\sum_{i=1}^{3} n_{i}^{b}=0$ implies that the line passing through $n_{1}^{b}$ and $n_{3}^{b}$ is parallel to the line passing through $A$ and the origin. These observations and elementary computations yield

$$
\delta=r\left(2 \sin (\pi / 8)-\frac{1}{2 \cos (\pi / 8)}\right), \quad\left|n_{1}^{a}-n_{1}^{b}\right|=r\left(\frac{1}{\cos (\pi / 8)}-2 \sin (\pi / 8)\right) .
$$

Denote by $X$ an arbitrary vector of the segment connecting $n_{1}^{a}$ and $n_{1}^{b}$ and let $x:=|A-X| \in$ $[\delta, l-\delta]$. If $Y$ (resp. $Z$ ) is a vector belonging to the segment connecting $n_{2}^{a}$ and $n_{2}^{b}$ (resp. $n_{3}^{a}$ and $n_{3}^{b}$ ) the condition $X+Y+Z=0$ implies

$$
y=y(x)=\frac{\delta}{l-2 \delta}(-x+l-\delta), \quad z=z(x)=\frac{\delta}{l-2 \delta}(x-\delta),
$$

where $y:=\left|n_{2}^{b}-Y\right|$ and $z:=\left|n_{3}^{a}-Z\right|$ (see Figure 1 ).

REMARK 5.2 Since in two dimensions $N_{\min }$ is unique and its values are fixed (up to sign) at the three vertices of the partition different from $q$ (where it equals $A, D, C$, see Figure 3), it follows that the triplet of the values of $N_{\min }$ at $q$ uniquely determines $N_{\min }$ on $\Sigma_{1}, \Sigma_{2}, \Sigma_{3}$, simply by linear interpolation.

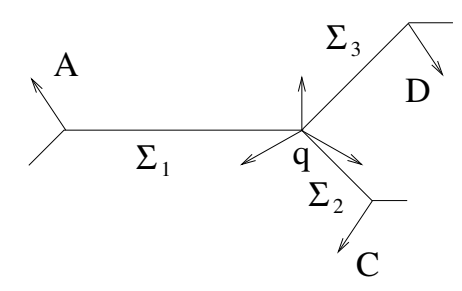

FIG. 3. The values of any admissible vector field are fixed (up to sign) with values $A, D, C$ at the vertices of the partition different from $q$; we also plot an admissible triplet at $q$.

Thanks to Remark 5.2, we can rewrite the functional appearing on the right hand side of (34) as a function of the variables $x, y(x), z(x)$ which are in a one-to-one correspondence with triplets of values of $N_{\min }$ at $q$. An easy computation shows that, for a vector field $N$ which is linear on each $\Sigma_{i}$ and satisfies the required constraints, we have, for the configuration in Figure 3 ,

$$
\int_{T}\left(\operatorname{div}_{\tau} N\right)^{2} \mathrm{~d} \mathcal{P}_{\varphi}=\frac{x^{2}}{L_{1}} \varphi^{o}\left(v_{1}\right)+\frac{(l-y(x))^{2}}{L_{2}} \varphi^{o}\left(v_{2}\right)+\frac{(l-z(x))^{2}}{L_{3}} \varphi^{o}\left(v_{3}\right),
$$

where $v_{i}$ are unit normal vectors to $\Sigma_{i}, i=1,2,3$. Observing that $\varphi^{o}\left(v_{1}\right)=\varphi^{o}\left(v_{2}\right)=\varphi^{o}\left(v_{3}\right)=$ : $\varphi^{o}(\nu)$, and inserting relations (51) into 52, we reduce the problem of finding $N_{\min }$ in (34) to the problem

$$
\min _{x \in[\delta, l-\delta]} f(x), \quad f(x):=\alpha x^{2}+\beta x+\gamma,
$$


where

$$
\begin{aligned}
& \alpha=\frac{1}{L_{1}}+\frac{\delta^{2}}{(l-2 \delta)^{2}}\left(\frac{1}{L_{2}}+\frac{1}{L_{3}}\right)>0, \\
& \beta=\frac{2 l \delta}{l-2 \delta}\left(\frac{1}{L_{2}}-\frac{1}{L_{3}}\right)+\frac{2 \delta^{2}}{(l-2 \delta)^{2}}\left(\delta\left(\frac{1}{L_{2}}-\frac{1}{L_{3}}\right)-\frac{l}{L_{2}}\right), \\
& \gamma=l^{2}\left(\frac{1}{L_{2}}+\frac{1}{L_{3}}\right)+\frac{2 l \delta}{l-2 \delta}\left(\frac{\delta}{L_{3}}-\frac{l-\delta}{L_{2}}\right)+\frac{\delta^{2}}{(l-2 \delta)^{2}}\left(\frac{(l-\delta)^{2}}{L_{2}}+\frac{\delta^{2}}{L^{3}}\right),
\end{aligned}
$$

and

$$
\frac{1}{\varphi^{o}(v)} \int_{T}\left(\operatorname{div}_{\tau} N_{\min }\right)^{2} \mathrm{~d} \mathcal{P}_{\varphi}=\min _{x \in[\delta, l-\delta]} f(x) .
$$

Let us denote by $x_{\min } \in[\delta, l-\delta]$ the minimum point in $[53$.

Recalling Definition 5.1, we observe that the condition

$$
\left.x_{\min } \in\right] \delta, l-\delta[
$$

is equivalent to the stability of $T$ (stability in the sense of Definition 5.1 can always be expressed through an inclusion relation similar to (55), for any polygonal Wulff shape and any admissible triple junction).

If $T$ is stable then no formation of new edges from $q$ is expected during the flow (for short times); if $T$ is unstable and, in addition, $f^{\prime}\left(x_{\min }\right)>0$ (resp. $\left.f^{\prime}\left(x_{\min }\right)<0\right)$ if $x_{\min }=\delta$ (resp. $x_{\min }=l-\delta$ ) then the appearance of a new edge starting from $q$ is expected (see Example 1).

We can now consider some special partitions $T$.

EXAMPLE 1 Let us consider the configuration $T$ of Figure 4 where we assume

$$
L_{1}=L_{2}=L_{3} .
$$

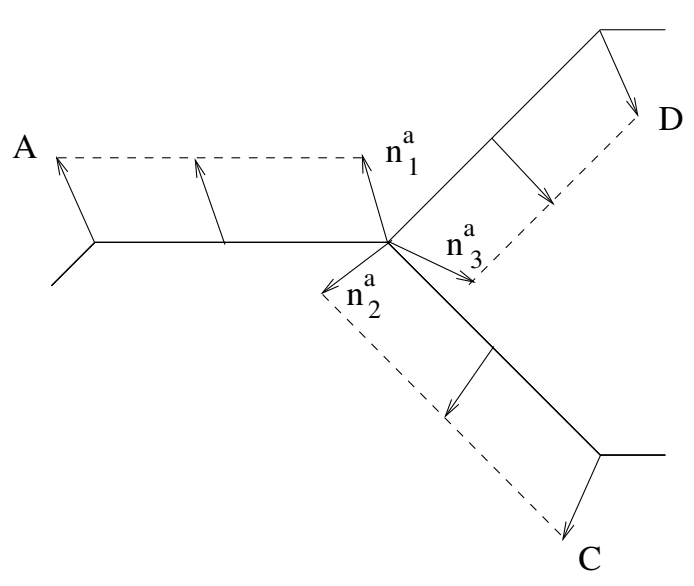

FIG. 4. Example $1\left(L_{1}=L_{2}=L_{3}\right)$. We plot the vector field $N_{\min }$. In this case $x_{\min }=\left|A-n_{1}^{a}\right|=\delta$. 


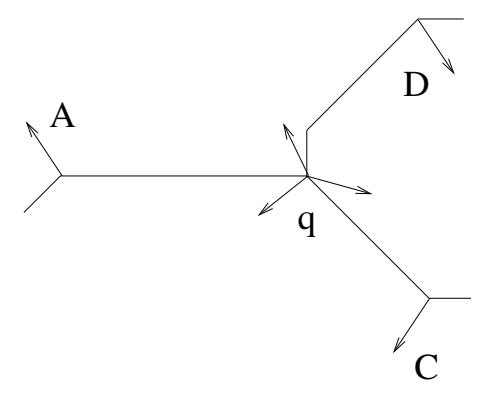

FIG. 5. Example 1: the appearance of the new vertical edge at $q$ in the evolution is due to the fact that $x_{\min }$ is not in the interior of the interval $[\delta, l-\delta]$. The edge is vertical, since $x_{\min }$ tends to be smaller than $\delta$ and the constraint $n_{\varphi} \in T^{o}\left(v_{\varphi}\right)$ cannot be violated.

In this case we have

$$
-\frac{\beta}{2 \alpha}=\frac{\delta^{2} l}{(l-2 \delta)^{2}+2 \delta^{2}}<\frac{l}{2}<l-\delta .
$$

To check that $-\beta /(2 \alpha) \geqslant \delta$ we have to verify that $(l-2 \delta)(l-3 \delta) \leqslant 0$; since $\delta<l / 2$, this reduces to checking whether $\delta \geqslant l / 3$. However, an elementary computation based on 50 yields $\delta<l / 3$. We conclude that $x_{\min }=\delta$ and $f^{\prime}\left(x_{\min }\right)>0$. The situation is depicted in Figure 4 the vector field $N_{\min }$ is the linear interpolation between $A$ and $n_{1}^{a}$ (recall that in this case $\left|A-n_{1}^{a}\right|=x_{\min }$ ) on $\Sigma_{1}$; similarly, it is the linear interpolation between $C$ and $n_{2}^{a}$ on $\Sigma_{2}$ and between $D$ and $n_{3}^{a}$ on $\Sigma_{3}$.

In this case the triple junction is unstable and a new edge is expected to appear in the subsequent evolution (cf. [28]). Our variational analysis allows us to determine a priori which new edge will appear. More precisely, a new vertical edge will be created as time flows, as in Figure 5 The reason why this should happen can be explained as follows: when minimizing the function $f$ in $(53)$, the value of $x_{\min }$ tends to decrease; if $x_{\min }<\delta$ the constraint $N_{\min } \in T^{o}\left(v_{\varphi}\right)$ cannot be satisfied on $\Sigma_{3}$ any more, unless a new vertical edge appears. On this new edge $N_{\min }$ will belong to a different edge of $\mathcal{W}_{\varphi}$, precisely the edge connecting $E$ and $n_{3}^{a}$ (see Figure 1 ).

EXAMPLE 2 If we let $L_{1}=+\infty$ and $L_{2}=L_{3}$ in Figure 3 , we get $\left.-\beta /(2 \alpha)=l / 2 \in\right] \delta, l-\delta[$. Therefore, if

$$
L_{2}=L_{3} \quad \text { and } \quad L_{1} \text { is sufficiently large, }
$$

we deduce that the minimum point $x_{\min }$ for $f$ in $(53)$ belongs to the interior of the interval $[\delta, l-\delta]$. Again, corresponding to this point, there are a unique triplet at $q$ and a unique vector field $N_{\min }$ defined on $T$ (by linear interpolation) whose tangential divergence is the $\varphi$-curvature of $T$. In this case the triple junction is stable.

EXAMPLE 3 Let us consider the configuration of Figure 6 In this case the function $f$ to be minimized in 53 is

$$
f(x)=\frac{x^{2}}{L_{1}}+\frac{(l-y(x))^{2}}{L_{2}}+\frac{z(x)^{2}}{L_{3}} .
$$

If $x \in] \delta, l-\delta[$ decreases then $y \in[0, l]$ increases and $z$ decreases. It follows that to minimize $f$ in 57 the value of $x_{\min }$ must be as small as possible, i.e. $x_{\min }=\delta$. Moreover, $f^{\prime}\left(x_{\min }\right)>0$. 


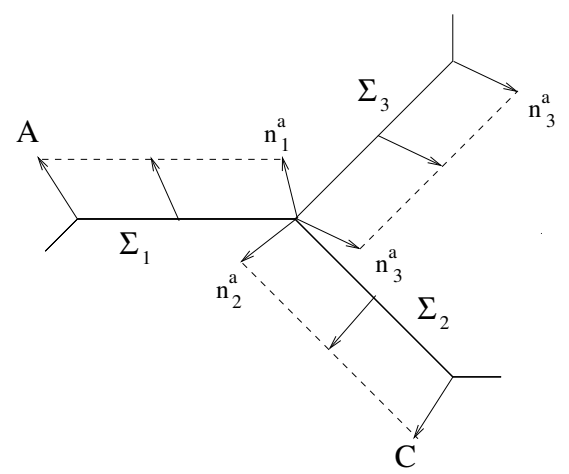

FIG. 6. Example 3: this triple junction is always unstable, for any choice of $L_{1}, L_{2}$ and $L_{3}$. Here we plot $N_{\text {min }}$ (linear interpolation on $\Sigma_{1}$ and $\Sigma_{2}$ and constant vector on $\Sigma_{3}$ ).

Therefore $\left(N_{\min }\right)_{\mid \Sigma_{i}}=n_{i}^{a}$ for any choice of $L_{i}, i=1,2,3$, and the triple junction is always unstable. In Figure 6 we plot $N_{\text {min }}$ (linear interpolation on $\Sigma_{1}$ and $\Sigma_{2}$, and constant vector on $\Sigma_{3}$ ).

EXAMPLE 4 Let us consider the partition $T$ of Figure 7 having two adjacent triple junctions $q_{1}$ and $q_{2}$. In this case we have two free variables $x_{1}, x_{2} \in[\delta, l-\delta]$, where $x_{i}:=\left|A-X\left(q_{i}\right)\right|$ and $X$ is an arbitrary admissible vector field on $\Sigma_{1}$. For a vector field which is linear on each $\Sigma_{i}$ and satisfies the required constraints we have

$$
\begin{aligned}
& \int_{T}\left(\operatorname{div}_{\tau} N\right)^{2} \mathrm{~d} \mathcal{P}_{\varphi} \\
& =\varphi^{o}(\nu)\left[\frac{\left(x_{1}-x_{2}\right)^{2}}{L_{1}}+\frac{\left(l-y\left(x_{1}\right)\right)^{2}}{L_{2}}+\frac{\left(l-z\left(x_{1}\right)\right)^{2}}{L_{3}}+\frac{\left(l-y\left(x_{2}\right)\right)^{2}}{L_{4}}+\frac{z\left(x_{2}\right)^{2}}{L_{5}}\right],
\end{aligned}
$$

where $y\left(x_{i}\right)=\frac{\delta}{l-2 \delta}\left(-x_{i}+l-\delta\right)$ and $z\left(x_{i}\right)=\frac{\delta}{l-2 \delta}\left(x_{i}-\delta\right), i=1$, 2. Inserting these relations in (58) we are reduced to the following minimum problem:

$$
\min _{\left(x_{1}, x_{2}\right) \in[\delta, l-\delta]^{2}} f\left(x_{1}, x_{2}\right), \quad f\left(x_{1}, x_{2}\right)=\alpha_{1} x_{1}^{2}+\alpha_{2} x_{2}^{2}+\alpha_{12} x_{1} x_{2}+\beta_{1} x_{1}+\beta_{2} x_{2}+\gamma,
$$

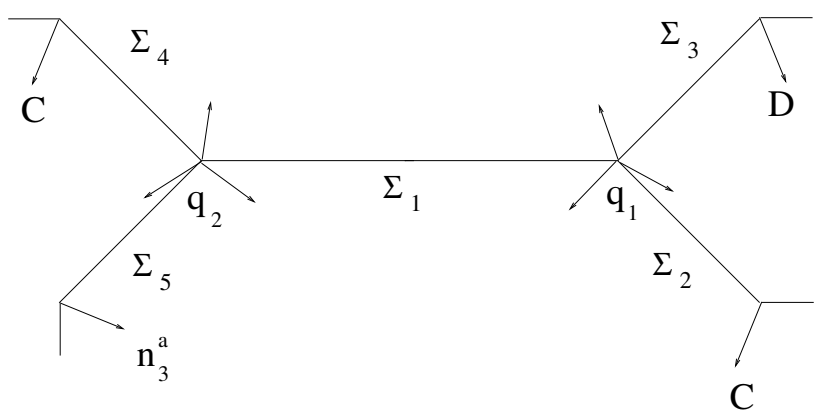

FIG. 7. Example 4: two adjacent triple junctions $q_{1}, q_{2}$; the function $f$ to be minimized is a quadratic polynomial in two variables. 
where

$$
\begin{aligned}
\alpha_{1}= & \frac{1}{L_{1}}+\frac{\delta^{2}}{(l-2 \delta)^{2}}\left(\frac{1}{L_{2}}+\frac{1}{L_{3}}\right)>0, \\
\alpha_{2}= & \frac{1}{L_{1}}+\frac{\delta^{2}}{(l-2 \delta)^{2}}\left(\frac{1}{L_{4}}+\frac{1}{L_{5}}\right)>0, \\
\alpha_{12}= & -\frac{2}{L_{1}}, \\
\beta_{1}= & \frac{2 \delta}{l-2 \delta}\left[l\left(\frac{1}{L_{2}}-\frac{1}{L_{3}}\right)+\frac{\delta}{l-2 \delta}\left(\delta\left(\frac{1}{L_{2}}-\frac{1}{L_{3}}\right)-\frac{l}{L_{2}}\right)\right], \\
\beta_{2}= & \frac{2 \delta}{l-2 \delta}\left[\frac{l}{L_{4}}+\frac{\delta}{l-2 \delta}\left(\delta\left(\frac{1}{L_{4}}-\frac{1}{L_{5}}\right)-\frac{l}{L_{4}}\right)\right], \\
\gamma= & l^{2}\left(\frac{1}{L_{2}}+\frac{1}{L_{3}}+\frac{1}{L_{4}}\right)+\frac{\delta^{4}}{(l-2 \delta)^{2}}\left(\frac{1}{L_{3}}+\frac{1}{L_{5}}\right)+\frac{\delta^{2}(l-\delta)^{2}}{(l-2 \delta)^{2}}\left(\frac{1}{L_{2}}+\frac{1}{L_{4}}\right) \\
& +\frac{2 l \delta}{l-2 \delta}\left(\frac{\delta}{L_{3}}+\frac{\delta-l}{L_{2}}+\frac{\delta-l}{L_{4}}\right) .
\end{aligned}
$$

The discussion on whether the configuration in Figure 7 is stable or unstable is now more complicated, in view of the dependence of the minimization problem $(59)$ on $L_{i}, i=1, \ldots, 5$, and each situation must be analyzed separately.

Observe that stability in the sense of Definition 5.1 is equivalent to

$$
\left.x_{\min }=\left(x_{1 \min }, x_{2 \min }\right) \in\right] \delta, l-\delta\left[^{2} .\right.
$$

If $T$ is not stable, then basically at least one of the two triple junctions is not stable; if in addition the gradient of $f$ points inside $[\delta, l-\delta]^{2}$, then the appearance of a new edge from one of the two triple junctions (or from both) is expected during the subsequent crystalline flow.

EXAMPLE 5 Let us consider the partition $T$ of Figure 8, which coincides with the partition of Figure 7 except for the way the network attaches to the edge $\Sigma_{3}$. Again, we have two free variables $x_{1}, x_{2} \in[\delta, l-\delta], x_{i}:=\left|A-X\left(q_{i}\right)\right|$ and $X$ an admissible vector field on $\Sigma_{1}$. In this case (58) is

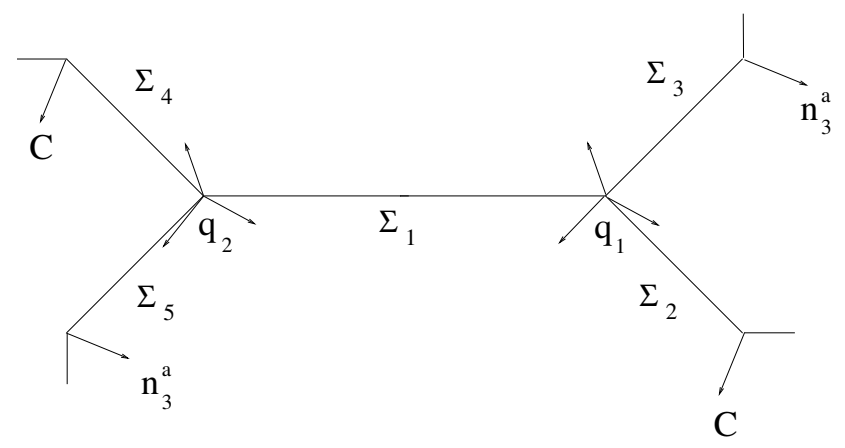

FIG. 8. Example 5: this configuration is always unstable, for any choice of $L_{i}, i=1, \ldots, 5$. Note that it consists of two unstable adjacent triple junctions. 
replaced by

$$
\begin{aligned}
\int_{T}\left(\operatorname{div}_{\tau} N\right)^{2} \mathrm{~d} \mathcal{P}_{\varphi} \\
\quad=\varphi^{o}(v)\left[\frac{\left(x_{1}-x_{2}\right)^{2}}{L_{1}}+\frac{\left(l-y\left(x_{1}\right)\right)^{2}}{L_{2}}+\frac{\left(z\left(x_{1}\right)\right)^{2}}{L_{3}}+\frac{\left(l-y\left(x_{2}\right)\right)^{2}}{L_{4}}+\frac{z\left(x_{2}\right)^{2}}{L_{5}}\right] .
\end{aligned}
$$

We observe that the second and third terms on the right hand side of (61) are strictly increasing in $\left.x_{1} \in\right] \delta, l-\delta\left[\right.$, and the fourth and fifth terms are strictly increasing in $\left.x_{2} \in\right] \delta, l-\delta$ [. Since the first term is zero when $x_{1}=x_{2}$, it follows that the minimum of the function in 61 is attained for $x_{1}=x_{2}=\delta$, therefore this configuration of two adjacent triple junctions is always unstable.

The instability of the configuration in Figure 8 could be related to the observation of Cahn and Kalonji [10], where they emphasize that neighbouring triple junctions must belong to different symmetry classes.

\subsection{A three-dimensional example}

In three dimensions the geometry of Lipschitz $\varphi$-regular partitions is more rich, and the junctions are lines instead of isolated points. Let us fix for simplicity the Wulff shape $\mathcal{W}_{\varphi}$ to be the cube of side $2 l$, i.e. $\varphi\left(\xi_{1}, \xi_{2}, \xi_{3}\right):=\frac{1}{l} \max \left(\left|\xi_{1}\right|,\left|\xi_{2}\right|,\left|\xi_{3}\right|\right)$. We want to construct Lipschitz $\varphi$-regular partitions. Let us consider a Lipschitz partition $\left\{E_{i}\right\}$ of $\mathbb{R}^{3}$ with the property that any surface $\partial E_{i} \cap \partial E_{j}=$ $\Sigma_{i j}$ is a union of rectangles parallel to some facet of $\partial \mathcal{W}_{\varphi}$, and two rectangles whose boundaries intersect belong to nonparallel planes (see for instance Figure 9, where a partition into five phases is depicted). We have the following observation.

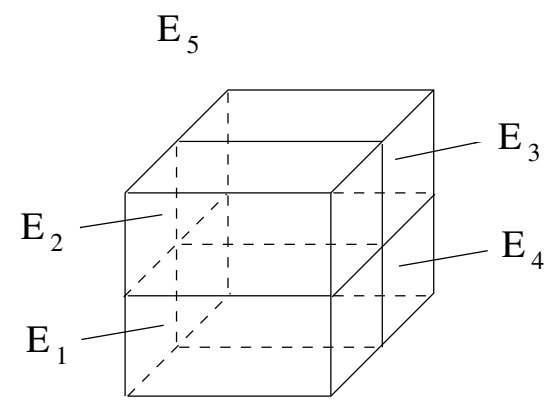

FIG. 9. A Lipschitz $\varphi$-regular partition of $\mathbb{R}^{3}$ into five solid phases when $\mathcal{W}_{\varphi}$ is a cube (the phase $E_{5}$ is the exterior of the cube).

REMARK 5.3 The partition $\left\{E_{i}\right\}$ is Lipschitz $\varphi$-regular, so that there exist vector fields $n_{\varphi}^{i j} \in$ $\operatorname{Lip}\left(\Sigma_{i j} ; \mathbb{R}^{3}\right)$ satisfying 31 .

Indeed, any $q \in \Gamma:=\bigcup_{i, j} \Sigma_{i j}$ has a neighbourhood $U_{q}$ such that $\Gamma \cap U_{q}$ coincides (up to translations and rotations) with one of the two sets of Figure 10. One can prove that the Lipschitz $\varphi$-regularity of the partition is a consequence of the Lipschitz $\varphi$-regularity of the two configurations 


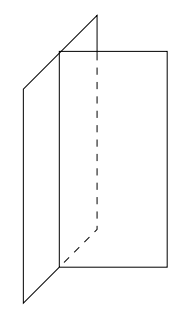

$\mathrm{T}_{1}$

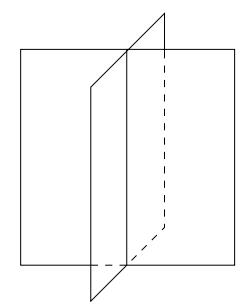

$\mathrm{T}_{2}$

FIG. 10. The local geometry of $\Gamma$ for a Lipschitz $\varphi$-regular partition.

$T_{1}, T_{2}$ in Figure 10 By considering horizontal sections of $T_{1}, T_{2}$, the proof of the Lipschitz $\varphi$ regularity can be reduced to a two-dimensional problem, i.e., to the proof of the Lipschitz $\widetilde{\varphi}$ regularity of $\widetilde{T}_{1}$ and $\widetilde{T}_{2}$, where $\{\widetilde{\varphi} \leqslant 1\}=\widetilde{\mathcal{W}}_{\varphi}$ is the horizontal section of the cube $\mathcal{W}_{\varphi}$ (that is, the square of side $2 l$ centered at the origin, see Figure 11 . Proving the Lipschitz $\widetilde{\varphi}$-regularity of $\widetilde{T}_{1}$ and $\widetilde{T}_{2}$ is then the analog of proving the Lipschitz regularity of the set in Figure 2 , with the octagon replaced by the square (see Figure 11 .

The first two assertions of the remark below follow from the fact that when we compute the Euler equation of the functional in [34], any vector field with constant divergence on each rectangle is a critical point, hence it is a minimizer since the functional is convex. The third assertion is a consequence of [2, Lemma 5.1].

REMARK 5.4 Any vector field minimizing [34] has constant divergence on each rectangle of $\Sigma_{i j}$. Conversely, assume that there exists a vector field $N \in H_{v, \varphi}^{\mathrm{div}}\left(T ; \mathbb{R}^{3}\right)$ satisfying 47 and such that $\operatorname{div}_{\tau} N$ is constant on each rectangle of $\Sigma_{i j}$. Then $N$ is a solution of the minimum problem on the right hand side of (34). Finally, there exists a minimizer $N_{\min }$ of (34) such that, for any rectangle $R$ of $T$, the normal trace $\left[N_{\min \mid R}, \widetilde{v}_{R}\right]$ is constant on any edge $e \subset \partial R$, where $\widetilde{v}_{R}$ is the euclidean unit normal to $\partial R$ pointing outwards from $R$ (in the plane of $R$ ).

Notice that the admissible triplet of vectors for the configuration $\widetilde{T}_{1}$ in Figure 11 is unique, while there are infinitely many admissible quadruplets for the configuration $\widetilde{T}_{2}$.

Observe also that no matching condition is required for the Lipschitz $\varphi$-regularity in three dimensions on zero-dimensional singular sets, i.e. we do not have to impose any condition on the isolated points which are intersections of segments belonging to $\Gamma$.

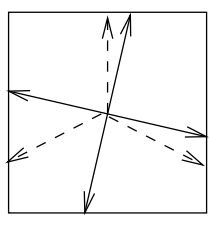

$\widetilde{\mathrm{w}}_{\phi}$

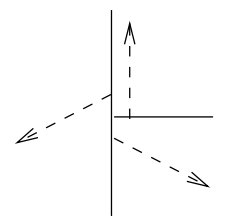

$\widetilde{\mathrm{T}}_{1}$

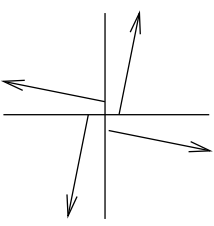

$\widetilde{\mathrm{T}}_{2}$

FIG. 11. Two Lipschitz regular partitions $\widetilde{T}_{1}$ and $\widetilde{T}_{2}$ (trijunction and quadrijunction) of $\mathbb{R}^{2}$ with respect to the metric whose unit ball $\widetilde{\mathcal{W}}_{\varphi}$ is the horizontal section of $\mathcal{W}_{\varphi}$. 
Under our assumptions on the partition $\left\{E_{i}\right\}$, it is possible to compute its crystalline mean curvature. As we shall see from (62) and $(63)$, we can equivalently rewrite the minimum problem in (34) as the constrained minimization of a quadratic polynomial depending only on a finite number of variables.

Let $N \in H_{v, \varphi}^{\text {div }}\left(T ; \mathbb{R}^{3}\right)$ be any vector field with constant divergence on each rectangle $R$ of $\Sigma_{i j}$, and having constant normal trace $\left[N_{\mid R}, \widetilde{v}_{R}\right]$ on each edge $e \subset \partial R$. We define $x_{e}^{i j}:=\left[N_{\mid R}, \widetilde{v}_{R}\right]_{\mid e} \in$ $[-l, l]$.

Notice that if $e$ does not belong to $\Gamma$ (as for instance the upper horizontal frontal edge in Figure 9), the quantity $x_{e}^{i j}$ is determined by the geometry of the partition (and is equal to $l$ or $-l$ ).

Condition (47) reduces to the linear system

$$
\sum_{R}\left(x_{e}^{i j} \tilde{\nu}_{R}+n_{\varphi}^{i j} \cdot v_{R} v_{R}\right)^{\partial R}=0 \quad \forall e \subset \Gamma,
$$

where the sum is over all pairs $R \subseteq \Sigma_{i j}$ such that $R \supset e$, and $v_{R}$ denotes a euclidean unit normal to $R$.

Recalling the divergence theorem and the fact that $N$ has constant divergence on each rectangle, we see that the function to be minimized is now the quadratic polynomial

$$
\int_{T}\left(\operatorname{div}_{\tau} N\right)^{2} \mathrm{~d} \mathcal{P}_{\varphi}=\varphi^{o}(v) \sum_{i j} \sum_{R \subseteq \Sigma_{i j}} \frac{1}{\mathcal{H}^{2}(R)}\left(\sum_{e \subset \partial R} \mathcal{H}^{1}(e) x_{e}^{i j}\right)^{2}
$$

in the variables $x_{e}^{i j}$, under the constraint 62. Finally, observe that the stability condition now reads $\left.x_{e}^{i j} \in\right]-l, l[$ for any edge $e \subset \Gamma$.

\section{REFERENCES}

1. Anzellotti, G. Pairings between measures and bounded functions and compensated compactness. Ann. Mat. Pura Appl. (4) 135 (1983), 293-318. Zbl 0572.46023 MR 85m:46042

2. Bellettini, G., Novaga, M., \& Paolini, M. Facet-breaking for three-dimensional crystals evolving by mean curvature. Interfaces Free Bound. 1 (1999), 39-55. Zbl 0934.49023 MR 1865105

3. Bellettini, G., Novaga, M., \& Paolini, M. On a crystalline variational problem, part I: first variation and global $L^{\infty}$-regularity. Arch. Ration. Mech. Anal. 157 (2001), 165-191. Zbl 0976.58016 MR 2002c:49072a

4. Bellettini, G., Novaga, M., \& Paolini, M. On a crystalline variational problem, part II: $B V$ regularity and structure of minimizers on facets. Arch. Ration. Mech. Anal. 157 (2001), 193-217. Zbl 0976.58017 MR 2002c:49072b

5. Bellettini, G. \& Novaga, M. Evolution of crystalline networks. In preparation.

6. Bellettini, G. \& Paolini, M. Anisotropic motion by mean curvature in the context of Finsler geometry. Hokkaido Math. J. 25 (1996), 537-566. Zbl 0873.53011 MR 97i:5307

7. Bellettini, G., Paolini, M., and Venturini, S. Some results on surface measures in calculus of variations. Ann. Mat. Pura Appl. (4) 170 (1996), 329-357. Zbl 0890.49020 MR 98e:49097

8. CAHN, J. W. Stability, microstructural evolution, grain growth, and coarsening in a two-dimensional two-phase microstructure. Acta Metall. Mater. 39 (1991), 2189-2199.

9. CAhn, J. W., Handwerker, C. A., \& TAYlor, J. E. Geometric models of crystal growth. Acta Metall. Mater. 40 (1992), 1443-1474. 
10. Cahn, J. W. \& Kalonji, G. Symmetries of grain boundary trijunctions. J. Phys. Chem. Solids 55 (1994), $1017-1022$.

11. Cahn, J. W. \& Van Vleck, E. Quadrijunctions do not stop two-dimensional grain growth. Scripta Mater. 34 (1996), 909-912.

12. Federer, H. Geometric Measure Theory. Springer, Berlin (1969). Zbl 0176.00801 MR 41 \#1976

13. Garcke, H. \& Nestler, B. A mathematical model for grain growth in thin metallic films. Math. Models Methods Appl. Sci. 10 (2000), 895-921. Zbl pre0188273 MR 2001b:74041

14. Garcke, H., Nestler, B., \& Stoth, B. A multiphase field concept: Numerical simulations of moving phase boundaries and multiple junctions. SIAM J. Appl. Math. 60 (1999), 295-315. Zbl 0942.35095 MR 2000j:74008

15. Giga, M. H. \& GigA, Y. Evolving graphs by singular weighted curvature. Arch. Ration. Mech. Anal. 141 (1998), 117-198. Zbl 0896.35069 MR 99j:35118

16. Giga, Y., Gurtin, M. E., \& Matias, J. On the dynamics of crystalline motion. Japan J. Indust. Appl. Math. 15 (1998), 7-50. MR 99h:73008

17. Herring, C. Surface tension as a motivation for sintering. The Physics of Powder Metallurgy, McGrawHill, New York (1951), 143-179.

18. Herring, C. The use of classical macroscopic concepts in surface energy problems. Structure and Properties of Solid Surfaces. Univ. of Chicago Press, Chicago (1952).

19. Hoffman, D. W. \& CAHn, J. W. A vector thermodynamics for anisotropic surfaces I. Fundamentals and application to plane surface junctions. Surf. Sci. 31 (1972), 368-388.

20. IKOTA, R. \& YANAGIDA, E. A stability criterion for stationary curves to the curvature driven motion with a triple junction. Preprint (2002).

21. Kinderlehrer, D., LiU, C., Manolache, F., \& TA'Asan, S. Remarks about analysis and simulation of grain boundary systems. Grain Growth in Polycrystalline Materials III, TMS (1998), 437-442.

22. LAWlor, G. \& Morgan, F. Paired calibrations applied to soap films, immiscible fluids, and surfaces or networks minimizing other norms. Pacific J. Math. 166 (1994), 55-83. Zbl 0830.49028 MR 95i:58051

23. Mullins, W. Two-dimensional motion of idealized grain boundaries. J. Appl. Phys. 27 (1956), 900-904. MR 17,1252

24. TAYloR, J. E. Crystalline variational problems. Bull. Amer. Math. Soc. 84 (1978), 568-588. Zbl 0392.49022 MR 58 \#12649

25. TAYlOR, J. E. Constructions and conjectures in crystalline nondifferential geometry. Differential Geometry. A Symposium in honour of Manfredo Do Carmo, Longman Sci. Tech. (1991), 321-336. Zbl 0725.53011 MR 93e:49004

26. TAYloR, J. E. II-mean curvature and weighted mean curvature. Acta Metall. Mater. 40 (1992), 14751485.

27. TAYlor, J. E. Motion of curves by crystalline curvature, including triple junctions and boundary points. Differential Geometry: Partial Differential Equations on Manifolds (Los Angeles, CA, 1990), Proc. Sympos. Pure Math. 54, Part 1, Amer. Math. Soc. (1993), 417-438. Zbl 0823.49028 MR 94c:53012

28. TAYlor, J. E. A variational approach to crystalline triple-junction motion. J. Statist. Phys. 95 (1999), 1221-1244. Zbl0952.74015 MR 2000i:74073 